Article

\title{
Conservation Status of Brachycephalus Toadlets (Anura: Brachycephalidae) from the Brazilian Atlantic Rainforest
}

\author{
Marcos R. Bornschein 1,2,*, Marcio R. Pie ${ }^{2,3}$ and Larissa Teixeira ${ }^{1}$ \\ 1 Instituto de Biociências, Universidade Estadual Paulista (UNESP), Praça Infante Dom Henrique s/no, \\ São Vicente, São Paulo, CEP 11330-900, Brazil \\ 2 Mater Natura-Instituto de Estudos Ambientais, Rua Lamenha Lins 1080, \\ Curitiba, Paraná, CEP 80250-020, Brazil \\ 3 Departamento de Zoologia, Universidade Federal do Paraná, Curitiba, Paraná, CEP 81531-980, Brazil \\ * Correspondence: bornschein.marcao@gmail.com or marcos.bornschein@unesp.br; Tel.: +55 13-98156-9582
}

Received: 1 July 2019; Accepted: 23 August 2019; Published: 27 August 2019

check for updates

\begin{abstract}
The number of described anurans has increased continuously, with many newly described species determined to be at risk. Most of these new species inhabit hotspots and are under threat of habitat loss, such as Brachycephalus, a genus of small toadlets that inhabits the litter of the Brazilian Atlantic Rainforest. Of 36 known species, 22 were described in the last decade, but only 11 have been assessed according to the IUCN Red List categories, with just one currently listed as Critically Endangered. All available data on occurrence, distribution, density, and threats to Brachycephalus were reviewed. The species extent of occurrence was estimated using the Minimum Convex Polygon method for species with three or more records and by delimiting continuous areas within the altitudinal range of species with up to two records. These data were integrated to assess the conservation status according to the IUCN criteria. Six species have been evaluated as Critically Endangered, five as Endangered, 10 as Vulnerable, five as Least Concern, and 10 as Data Deficient. Deforestation was the most common threat to imperiled Brachycephalus species. The official recognition of these categories might be more readily adopted if the microendemic nature of their geographical distribution is taken into account.
\end{abstract}

Keywords: deforestation; timber harvest; fire; invasion of exotic plants; conservation; public policy; protected areas; critically endangered; data deficient

\section{Introduction}

Frogs and toads (Anura) comprise more than 7000 species worldwide [1]. Special attention has been given to this group due to the large number of new species described each year as well as due to the increasing number of endangered species [2,3]. According to the IUCN Red List criteria [4,5], there are 1825 species of anurans at risk of extinction (25\% of all species), making Anura the vertebrate order with the highest proportion of endangered species [5]. Since 1980, there have been records of a rapid population decline of nearly 450 anuran species [6-8]. The decline of these species can be mainly attributed to habitat loss and pathogens, such as chytrid fungi and Ranavirus [6,7,9-11]. Recently, Ranavirus has been reported in natural populations of frogs in South America, but the effects in wild anuran populations are still unknown [11]. Unlike Ranavirus, chytrid fungi (Batrachochytrium dendrobatidis) has been commonly reported as a cause of population decline in high altitude locations in Costa Rica and Panama [9]. Due to the rapid rate of the description of a new species, the proportion of endangered species, and sensitivity, Anura is the priority order for 
a conservation assessment, particularly in countries with a high level of deforestation, such as in Brazil [3].

The Atlantic Rainforest, a biodiversity hotspot [12], is the largest in area after the Amazon forest, with its original extent covering more than 1.3 million $\mathrm{km}^{2}[13,14]$. It is located on the eastern coast of South America, stretching from northeastern to southern Brazil, with inland extensions to the east of Paraguay, northeast of Argentina, and central Brazil. This biome has been experiencing massive habitat loss due to agricultural expansion, urbanization, and historic loss of natural habitats [15]. Currently, only $28 \%$ of the original extent remains if secondary forests and forests affected by the edge effects are included [15]. The Atlantic Rainforest houses nearly 2500 species of vertebrates, including 550 anurans, of which 323 are endemic $(63 \%)$ and 15 are currently considered to be threatened by extinction $[1,5,16]$.

The genus Brachycephalus (Fitzinger, 1826) is endemic to the Atlantic Rainforest and includes small (less than $2.5 \mathrm{~cm}$ in snout-vent length) diurnal toadlets with a reduced number of digits, bright colors, neurotoxins in the skin, and direct development, and they live in leaf litter, specifically that of montane forests [17-23]. There are currently 36 recognized species of Brachycephalus [1], of which 22 have been described in the last decade [1]. Most have extremely restricted geographical distributions of less than 100 ha $[12,24,25]$. Brachycephalus is divided into three phenetic groups [26], two of which (B. ephippiumsi and B. pernix groups) are montane with few records at lower altitudes, whereas the remaining group (B. didactylus group) includes more ecologically plastic species that occur from the sea level up to high altitudes [23,27]. The dependence on a colder climate and isolation in the mountains as sky islands have been hypothesized as the reason that montane groups have diverged into so many species (19 of B. pernix and 12 of B. ephippiumsi groups), whereas the B. didactylus group includes only four species $[23,28,29]$. Another species (B. atelopoide) cannot be compared to any of the groups due to the unavailability of the holotype [23,30].

Species descriptions of Brachycephalus have not been accompanied by corresponding assessments of the conservation status. Only 11 species have been assessed for the IUCN Red List to date [31-41]: eight as Data Deficient (DD) and three as Least Concern (LC). The Ministério do Meio Ambiente (MMA, the Ministry of the Environment of the Brazilian government) evaluated only four species and categorized one as Critically Endangered (CR), two as DD, and one as Near Threatened (NT) [42-45]. The absence of conservation status assessments of most species and the evaluation of some of them as DD highlight the need for a comprehensive effort to assess the risk of extinction of the Brachycephalus species, most notably the microendemic taxa found in the B. pernix and B. ephippiumsi species groups (sensu [26]). Species evaluated as DD should be prioritized to generate enough data to properly classify them into a conservation category $[46,47]$.

One way to direct effective initiatives for conservation species is through a prior assessment of their conservation status [3]. There is a widely adopted IUCN methodology for proposing a conservation status [3], which serves an important role in allowing for comparisons and for classifying conservation actions as well the proposition of public policies. The objectives of the study were (1) to review data on occurrence, altitudinal distribution, density, and threats to the Brachycephalus species, (2) to compile new data from the literature and unpublished observations, (3) to generate systematized data on geographic distribution, population sizes, and threats to place them into IUCN conservation categories, and (4) to discuss conservation priorities and future management actions.

\section{Material and Methods}

All available occurrence records of Brachycephalus spp. were compiled from the literature up to the time of compilation (June 2019). The data encompassed toponymy, species identification, geographical coordinates of the occurrence record, and altitude of the corresponding site. Data on altitudinal range were also considered when available. The process began with the latest compilation of locality and altitude data for Brachycephalus provided by Bornschein et al. [23], and the same selection criteria were adopted for subsequent records. For example, those associated with precise localities were retained, 
and records that included only municipality names as occurrence information were discarded. Finally, the authors' previously unpublished data were included.

Occurrence records were plotted using Google Earth Pro v. 7.1.4.1529 and connected to form a polygon using the Minimum Convex Polygon approach (MCP; [48]) with modifications suggested by Reinert et al. [49] and adopted by Bornschein et al. [23]. These modifications allow for the exclusion of inappropriate habitats, such as bodies of water, pastures, silvicultures, urban areas, rock areas, and/or forest areas, beyond the altitudinal range of occurrence of the species.

Polygon delimitation required three or more occurrence records. For species with one or two records, polygons encompassing the altitudinal range of the species were created [23]. A continuous topography inside the polygon was considered a location (sensu IUCN and as IUCN [48]) that could potentially contain one or more records of a given species. The topography was considered discontinuous if it was isolated by altitudes beyond the altitudinal range of the respective species.

The MCP and altitudinal polygons were measured using GEPath v. 1.4.5 to obtain the extent of occurrence (EO; IUCN [48]; see also $[23,25,50]$ ) of each species. Because some species have such reduced EO, they could potentially also be ranked by area of occupancy (AO), although AO was not measured in this study; however, species with less than 1000 ha of EO could also be categorized based on the criterion of an AO of less than 1,000 ha (criteria B2, for CR [48]) as well as species with an AO less than 50,000 ha (criteria B2, for EN [48]) because AO is always smaller than EO and is located within the EO polygon [48].

Population size was inferred for each species based on the estimates of area in $\mathrm{m}^{2}$ inhabited by one individual compiled by Bornschein et al. [24]. Based on estimates of the number of calling males [24], a sex ratio of one female per male [24] was assumed. In cases with distinct estimates of densities per species [24], the mean density was used. The mean area in which one individual per species can be found and its respective EO was then used to calculate the population size.

Data on EO, number of locations, population size, and threats of the species were integrated to evaluate and to categorize its conservation status according to the IUCN Red List and Criteria [48]. For the recognition of threats, data from the literature, personal field experience of the authors collected in the EO of 29 species, and information on land use, forest quality, and trends of deforestation over the previous 10 years were considered. For temporal trends in land use, the time series of satellite images of Google Earth Pro v. 7.1.4.1529 was analyzed.

In the treatment of the data in relation to the IUCN criteria, the flow chart presented in Figure 1 was used. Six pathways were developed beginning with the evaluation of the number of localities (one to two; three or more). If the species had up to two recorded localities, its altitudinal range was calculated. If an altitudinal range was not associated with the record, this prevented creating a polygon and estimating the EO. The species was then considered DD (pathway 3 of Figure 1). If the records were associated with altitudinal range, an EO was created based on the lower and upper altitudinal limits. It was not always possible to infer the $\mathrm{EO}$ without encompassing inland areas far west of the record and outside the assumed natural range, sometimes nearly reaching Argentina, which is clearly unrealistic. In these situations, the species were considered DD (pathway 2 of Figure 1). When there were up to two records associated with an altitudinal range that encompassed a realistic polygon for EO (as indicated), the status of the species was evaluated (pathway 1 of Figure 1). Further pathways related to the procedure can be observed in Figure 1. 


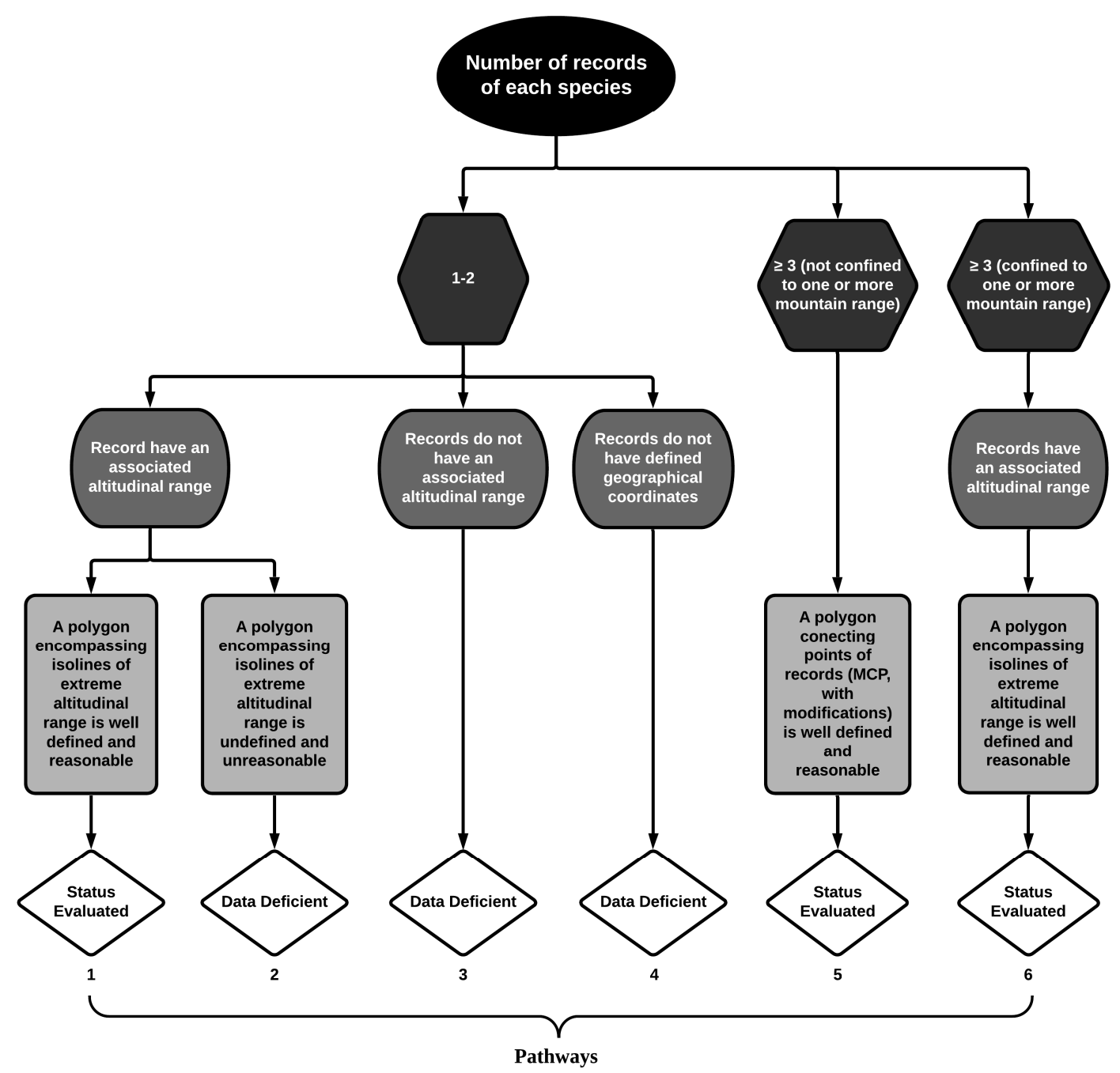

Figure 1. Flow chart indicating the approach to creating polygons of the extent of occurrence to compare the results with IUCN's species extinction risk classification criteria [48].

\section{Results}

A total of 185 locality records representing all 36 currently recognized Brachycephalus species in addition to 32 Brachycephalus sp. were generated (Table 1). An unidentified Brachycephalus species represented one between two described species that could not be adequately identified (i.e., old museum material collected before certain species were described) as well as new species awaiting formal description. Hereafter, only the described species are analyzed, leaving any evaluations to their own descriptors. The EO for 26 species (Table 1) was estimated, comprising several highly restricted EOs as well as larger ones: 23.8 ha for B. fuscolineatus, 37.4 ha for B. coloratus, 38.8 ha for B. boticario, 41.4 ha for B. tridactylus, 56.8 ha for B. mirissimus (all from the B. pernix group), 143,325.0 ha for B. hermogenesi, 702,983.4 ha for B. didactylus, 3,021,786.1 ha for B. sulfuratus (B. didactylus group), and $1,792,535.1$ ha for B. ephippiumsi (B. ephippiumsi group). The population sizes of eight species (Table 1) was also estimated. All were highly abundant with population sizes ranging from 78,344 individuals for B. mirissimus and 302,178,610 individuals for B. sulfuratus. 
Table 1. Locality records of Brachycephalus.

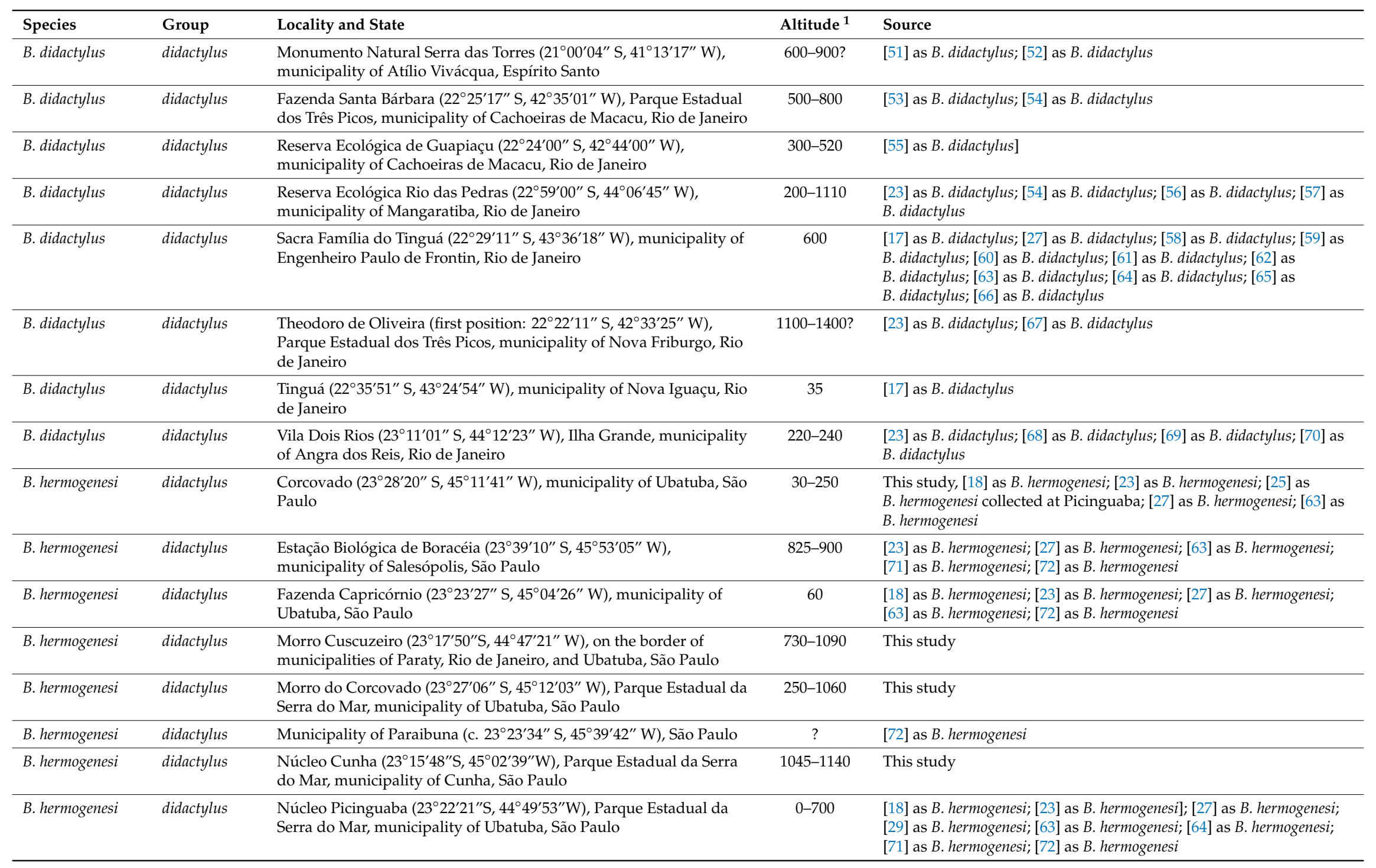


Table 1. Cont

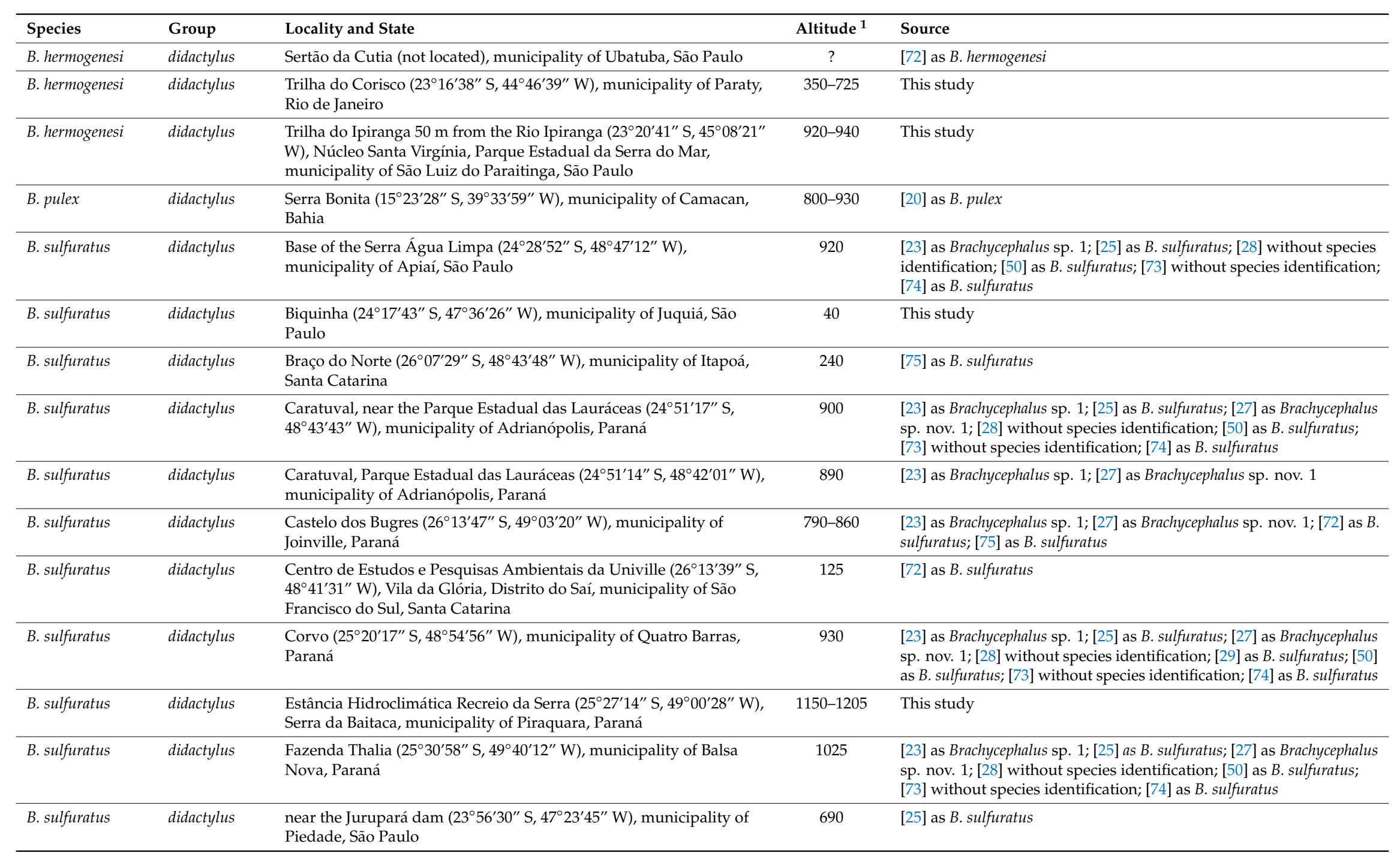


Table 1. Cont

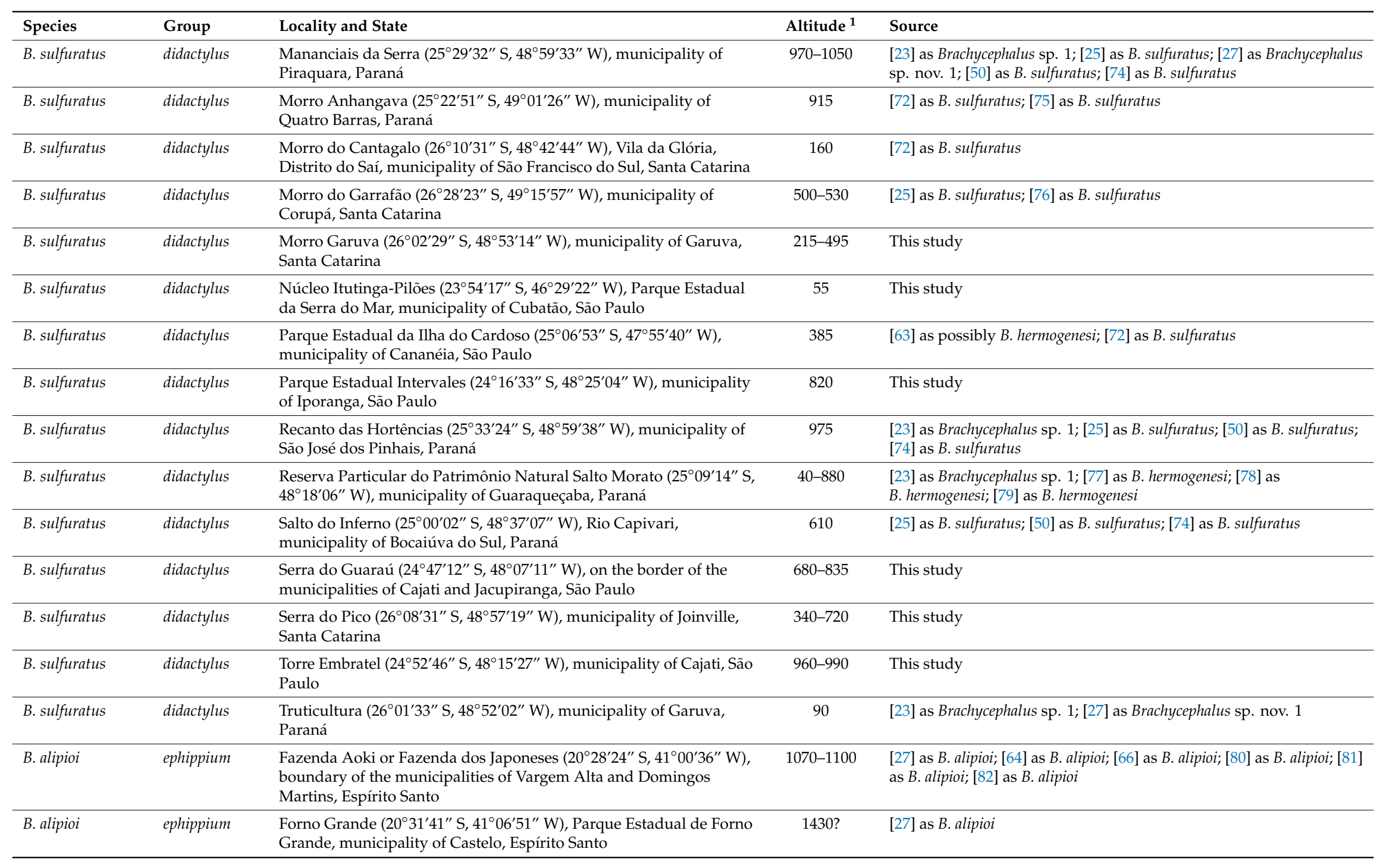


Table 1. Cont

\begin{tabular}{|c|c|c|c|c|}
\hline Species & Group & Locality and State & Altitude $^{1}$ & Source \\
\hline B. alipioi & ephippium & $\begin{array}{l}\text { Alto Castelinho }\left(20^{\circ} 30^{\prime} 34^{\prime \prime} \mathrm{S}, 41^{\circ} 00^{\prime} 33^{\prime \prime} \mathrm{W}\right) \text {, municipality of Vargem } \\
\text { Alta, Espírito Santo }\end{array}$ & 1100 & This study, [25] as B. alipioi \\
\hline B. bufonoides & ephippium & $\begin{array}{l}\text { Serra de Macaé }\left(22^{\circ} 18^{\prime} 02^{\prime \prime} \mathrm{S}, 42^{\circ} 18^{\prime} 20^{\prime \prime} \mathrm{W}\right) \text {, municipality of Nova } \\
\text { Friburgo, Rio de Janeiro }\end{array}$ & $1100 ?$ & $\begin{array}{l}\text { [30] as B. bufonoides; [66] as B. bufonoides; [83] as B. ephippiumsi } \\
\text { bufonoides }\end{array}$ \\
\hline B. crispus & ephippium & $\begin{array}{l}\text { Bacia B, Núcleo Cunha, Parque Estadual da Serra do Mar }\left(23^{\circ} 15^{\prime} 15^{\prime \prime}\right. \\
\text { S, } 45^{\circ} 01^{\prime} 58^{\prime \prime} \text { W), municipality of Cunha, São Paulo }\end{array}$ & $800-1190$ & This study, [84] as B. crispus \\
\hline B. darkside & ephippium & $\begin{array}{l}\text { Mata do Pai Inácio }\left(20^{\circ} 46^{\prime} 44^{\prime \prime} \mathrm{S}, 42^{\circ} 29^{\prime} 10^{\prime \prime} \mathrm{W}\right) \text {, Parque Estadual da } \\
\text { Serra do Brigadeiro, municipality Miradouro, Minas Gerais }\end{array}$ & 1340 & [66] as B. ephippiumsi; [85] as B. ephippiumsi; [86] as B. darkside \\
\hline B. ephippiumsi & ephippium & $\begin{array}{l}\text { Condomínio Ermida ( } 23^{\circ} 14^{\prime} 13^{\prime \prime} \text { S, } 46^{\circ} 58^{\prime} 52^{\prime \prime} \text { W), Serra do Japi, } \\
\text { municipality of Jundiaí, São Paulo }\end{array}$ & 1225 & [27] as B. ephippiumsi \\
\hline B. ephippiumsi & ephippium & $\begin{array}{l}\text { Hotel Fazenda Pé da Serra }\left(22^{\circ} 51^{\prime} 56^{\prime \prime} S, 45^{\circ} 31^{\prime} 40^{\prime \prime} \text { W), municipality }\right. \\
\text { of Pindamonhangaba, São Paulo }\end{array}$ & 700 & [27] as B. ephippiumsi \\
\hline B. ephippiumsi & ephippium & $\begin{array}{l}\text { Lago Azul }\left(22^{\circ} 27^{\prime} 23^{\prime \prime} \mathrm{S}, 44^{\circ} 36^{\prime} 34^{\prime \prime} \mathrm{W}\right) \text {, Parque Nacional do Itatiaia, } \\
\text { municipality of Itatiaia, Rio de Janeiro }\end{array}$ & 750 & [27] as B. ephippiumsi \\
\hline B. ephippiumsi & ephippium & $\begin{array}{l}\text { Maromba }\left(22^{\circ} 25^{\prime} 43^{\prime \prime} \mathrm{S}, 44^{\circ} 37^{\prime} 11^{\prime \prime} \mathrm{W}\right) \text {, Parque Nacional do Itatiaia, } \\
\text { municipality of Itatiaia, Rio de Janeiro }\end{array}$ & 1125 & [27] as B. ephippiumsi \\
\hline B. ephippiumsi & ephippium & $\begin{array}{l}\text { Monteiro Lobato }\left(22^{\circ} 57^{\prime} 07^{\prime \prime} \mathrm{S}, 45^{\circ} 50^{\prime} 20^{\prime \prime} \mathrm{W}\right) \text {, municipality of } \\
\text { Monteiro Lobato, São Paulo }\end{array}$ & 700 & [66] as B. ephippiumsi \\
\hline B. ephippiumsi & ephippium & $\begin{array}{l}\text { Observatório de Capricórnio }\left(22^{\circ} 53^{\prime} 54^{\prime \prime} \mathrm{S}, 46^{\circ} 49^{\prime} 01^{\prime \prime} \mathrm{W}\right) \text {, Serra das } \\
\text { Cabras, Joaquim Egídio District, boundary of the municipalities of } \\
\text { Campinas and Morungaba, São Paulo }\end{array}$ & 1085 & $\begin{array}{l}\text { [19] as B. ephippiumsi]; [27] as B. ephippiumsi; [66] as B. ephippiumsi; } \\
\text { [87] as B. ephippiumsi }\end{array}$ \\
\hline B. ephippiumsi & ephippium & $\begin{array}{l}\text { Parque Municipal de Itapetinga (Grota Funda) }\left(23^{\circ} 11^{\prime} 07^{\prime \prime} S, 46^{\circ} 31^{\prime} 47^{\prime \prime}\right. \\
\text { W), municipality of Atibaia, São Paulo }\end{array}$ & $900-1250$ & $\begin{array}{l}\text { [27] as B. ephippiumsi; [64] as B. ephippiumsi; [81] as B. ephippiumsi; [88 } \\
\text { as B. ephippiumsi; [89] as B. ephippiumsi }\end{array}$ \\
\hline B. ephippiumsi & ephippium & $\begin{array}{l}\text { Reserva Biológica da Serra do Japi }\left(23^{\circ} 17^{\prime} 07^{\prime \prime} \text { S, } 47^{\circ} 00^{\prime} 05^{\prime \prime} \text { W), Serra }\right. \\
\text { do Japi, boundary of the municipalities of Jundiaí and Cabreúva, São } \\
\text { Paulo }\end{array}$ & 1000 & $\begin{array}{l}\text { [27] as B. ephippiumsi; [64] as B. ephippiumsi; [66] as B. ephippiumsi; [90 } \\
\text { as B. ephippiumsi }\end{array}$ \\
\hline B. ephippiumsi & ephippium & $\begin{array}{l}\text { Reserva Ecológica do Trabiju }\left(22^{\circ} 48^{\prime} 01^{\prime \prime} \mathrm{S}, 45^{\circ} 32^{\prime} 03^{\prime \prime} \text { W), Trabiju, }\right. \\
\text { municipality of Pindamonhangaba, São Paulo }\end{array}$ & $1000 ?$ & [66] as B. ephippiumsi \\
\hline B. ephippiumsi & ephippium & $\begin{array}{l}\text { Reserva Pedra Branca }\left(22^{\circ} 56^{\prime} 22^{\prime \prime} \text { S, } 45^{\circ} 41^{\prime} 04^{\prime \prime} \text { W), municipality of }\right. \\
\text { Tremembé, São Paulo }\end{array}$ & $890 ?$ & [66] as B. ephippiumsi \\
\hline B. ephippiumsi & ephippium & $\begin{array}{l}\text { Santo Antônio do Pinhal }\left(22^{\circ} 49^{\prime} 28^{\prime \prime} \text { S, } 45^{\circ} 40^{\prime} 20^{\prime \prime} \text { W), municipality of }\right. \\
\text { Santo Antônio do Pinhal, São Paulo }\end{array}$ & 1080 & [66] as B. ephippiumsi \\
\hline
\end{tabular}


Table 1. Cont

\begin{tabular}{|c|c|c|c|c|}
\hline Species & Group & Locality and State & Altitude $^{1}$ & Source \\
\hline B. ephippiumsi & ephippium & $\begin{array}{l}\text { São Francisco Xavier }\left(22^{\circ} 53^{\prime} 44^{\prime \prime} \text { S, } 45^{\circ} 58^{\prime} 04^{\prime \prime} \text { W), municipality of São }\right. \\
\text { José dos Campos, São Paulo }\end{array}$ & 1000 & $\begin{array}{l}\text { [27] as B. ephippiumsi; [66] as B. ephippiumsi; [91] as B. ephippiumsi; [92 } \\
\text { as B. ephippiumsi }\end{array}$ \\
\hline B. ephippiumsi & ephippium & $\begin{array}{l}\text { Serra Negra }\left(21^{\circ} 57^{\prime} 28^{\prime \prime} \mathrm{S}, 43^{\circ} 47^{\prime} 20^{\prime \prime} \mathrm{W}\right) \text {, municipality of Santa } \\
\text { Bárbara do Monte Verde, Minas Gerais }\end{array}$ & $?$ & [23] as B. ephippiumsi; [65] as BMV MG2 \\
\hline B. ephippiumsi & ephippium & $\begin{array}{l}\text { Serra da Concórdia }\left(22^{\circ} 20^{\prime} 30^{\prime \prime} \mathrm{S}, 43^{\circ} 44^{\prime} 04^{\prime \prime} \mathrm{W}\right) \text {, Parque Estadual Serra } \\
\text { da Concórdia, Barão de Juparanã, municipality of Valença, Rio de } \\
\text { Janeiro }\end{array}$ & $900 ?$ & [66] as B. ephippiumsi \\
\hline B. ephippiumsi & ephippium & $\begin{array}{l}\text { Alto do Soberbo }\left(22^{\circ} 27^{\prime} 15^{\prime \prime} \mathrm{S}, 42^{\circ} 59^{\prime} 21^{\prime \prime} \mathrm{W}\right) \text {, municipality of } \\
\text { Teresópolis, Rio de Janeiro }\end{array}$ & 1250 & [66] as B. ephippiumsi \\
\hline B. ephippiumsi & ephippium & $\begin{array}{l}\text { Comary }\left(22^{\circ} 27^{\prime} 22^{\prime \prime} \mathrm{S}, 42^{\circ} 58^{\prime} 24^{\prime \prime} \mathrm{W}\right) \text {, municipality of Teresópolis, Rio } \\
\text { de Janeiro }\end{array}$ & 990 & [66] as B. ephippiumsi \\
\hline B. ephippiumsi & ephippium & $\begin{array}{l}\text { Floresta dos Macacos }\left(22^{\circ} 58^{\prime} 15^{\prime \prime} \mathrm{S}, 43^{\circ} 15^{\prime} 24^{\prime \prime} \mathrm{W}\right) \text {, municipality of Rio } \\
\text { de Janeiro, Rio de Janeiro }\end{array}$ & $450 ?$ & [66] as B. ephippiumsi \\
\hline B. ephippiumsi & ephippium & $\begin{array}{l}\text { Garrafão }\left(22^{\circ} 28^{\prime} 04^{\prime \prime} S, 43^{\circ} 01^{\prime} 52^{\prime \prime} \mathrm{W}\right) \text {, municipality of Guapimirim, } \\
\text { Rio de Janeiro }\end{array}$ & $1785 ?$ & [66] as B. ephippiumsi \\
\hline B. ephippiumsi & ephippium & $\begin{array}{l}\text { Pedra Branca }\left(22^{\circ} 55^{\prime} 55^{\prime \prime} \mathrm{S}, 43^{\circ} 28^{\prime} 23^{\prime \prime} \text { W), Serra da Pedra Branca, }\right. \\
\text { municipality of Rio de Janeiro, Rio de Janeiro }\end{array}$ & 1000 & [58] as B. ephippiumsi; [66] as B. ephippiumsi \\
\hline B. ephippiumsi & ephippium & $\begin{array}{l}\text { Represa do Rio Grande }\left(22^{\circ} 55^{\prime} 58^{\prime \prime} \text { S }, 43^{\circ} 26^{\prime} 36^{\prime \prime} \text { W), Parque Estadual }\right. \\
\text { da Pedra Branca, municipality of Rio de Janeiro, Rio de Janeiro }\end{array}$ & $150 ?$ & [27] as B. ephippiumsi; [66] as B. ephippiumsi \\
\hline B. ephippiumsi & ephippium & $\begin{array}{l}\text { Reserva Ecológica Rio das Pedras }\left(22^{\circ} 59^{\prime} 00^{\prime \prime} \mathrm{S}, 44^{\circ} 06^{\prime} 45^{\prime \prime} \mathrm{W}\right) \text {, } \\
\text { municipality of Mangaratiba, Rio de Janeiro }\end{array}$ & $200-1110$ & [56] as B. ephippiumsi \\
\hline B. ephippiumsi & ephippium & $\begin{array}{l}\text { Riacho Beija-flor }\left(22^{\circ} 27^{\prime} 04^{\prime \prime} \mathrm{S}, 43^{\circ} 00^{\prime} 04^{\prime \prime} \mathrm{W}\right) \text {, Parque Nacional da } \\
\text { Serra dos Orgãos, municipality of Teresópolis, Rio de Janeiro }\end{array}$ & 1195 & [27] as B. ephippiumsi \\
\hline B. ephippiumsi & ephippium & $\begin{array}{l}\text { Rocio District }\left(22^{\circ} 28^{\prime} 23^{\prime \prime} \mathrm{S}, 43^{\circ} 14^{\prime} 38^{\prime \prime} \mathrm{W}\right) \text {, municipality of Petrópolis, } \\
\text { Rio de Janeiro }\end{array}$ & 950 & [27] as B. ephippiumsi \\
\hline B. ephippiumsi & ephippium & $\begin{array}{l}\text { Serra do Tinguá }\left(22^{\circ} 35^{\prime} 31^{\prime \prime} \mathrm{S}, 43^{\circ} 28^{\prime} 16^{\prime \prime} \mathrm{W}\right) \text {, municipality of Nova } \\
\text { Iguaçu, Rio de Janeiro }\end{array}$ & $950 ?$ & [66] as B. ephippiumsi \\
\hline B. ephippiumsi & ephippium & $\begin{array}{l}\text { Vale da Revolta }\left(22^{\circ} 26^{\prime} 17^{\prime \prime} \mathrm{S}, 42^{\circ} 56^{\prime} 19^{\prime \prime} \mathrm{W}\right) \text {, municipality of } \\
\text { Teresópolis, Rio de Janeiro }\end{array}$ & 1035 & [66] as B. ephippiumsi \\
\hline B. ephippiumsi & ephippium & $\begin{array}{l}\text { Varginha }\left(22^{\circ} 24^{\prime} 34^{\prime \prime} S, 42^{\circ} 52^{\prime} 11^{\prime \prime} \mathrm{W}\right) \text {, municipality of Teresópolis, Rio } \\
\text { de Janeiro }\end{array}$ & $825 ?$ & [66] as B. ephippiumsi \\
\hline B. ephippiumsi & ephippium & $\begin{array}{l}\left.\text { Bonito ( } 22^{\circ} 42^{\prime} 51^{\prime \prime} \mathrm{S}, 44^{\circ} 34^{\prime} 39^{\prime \prime} \mathrm{W}\right) \text {, Serra da Bocaina, municipality of } \\
\text { São José do Barreiro, São Paulo }\end{array}$ & $1660 ?$ & [66] as B. ephippiumsi \\
\hline B. ephippiumsi & ephippium & $\begin{array}{l}\text { Estação Ecológica de Bananal }\left(22^{\circ} 48^{\prime} 05^{\prime \prime} \text { S, } 44^{\circ} 22^{\prime} 12^{\prime \prime} \text { W), Serra da }\right. \\
\text { Bocaina, municipality of Bananal, São Paulo }\end{array}$ & $1200 ?$ & [93] as B. ephippiumsi \\
\hline
\end{tabular}


Table 1. Cont

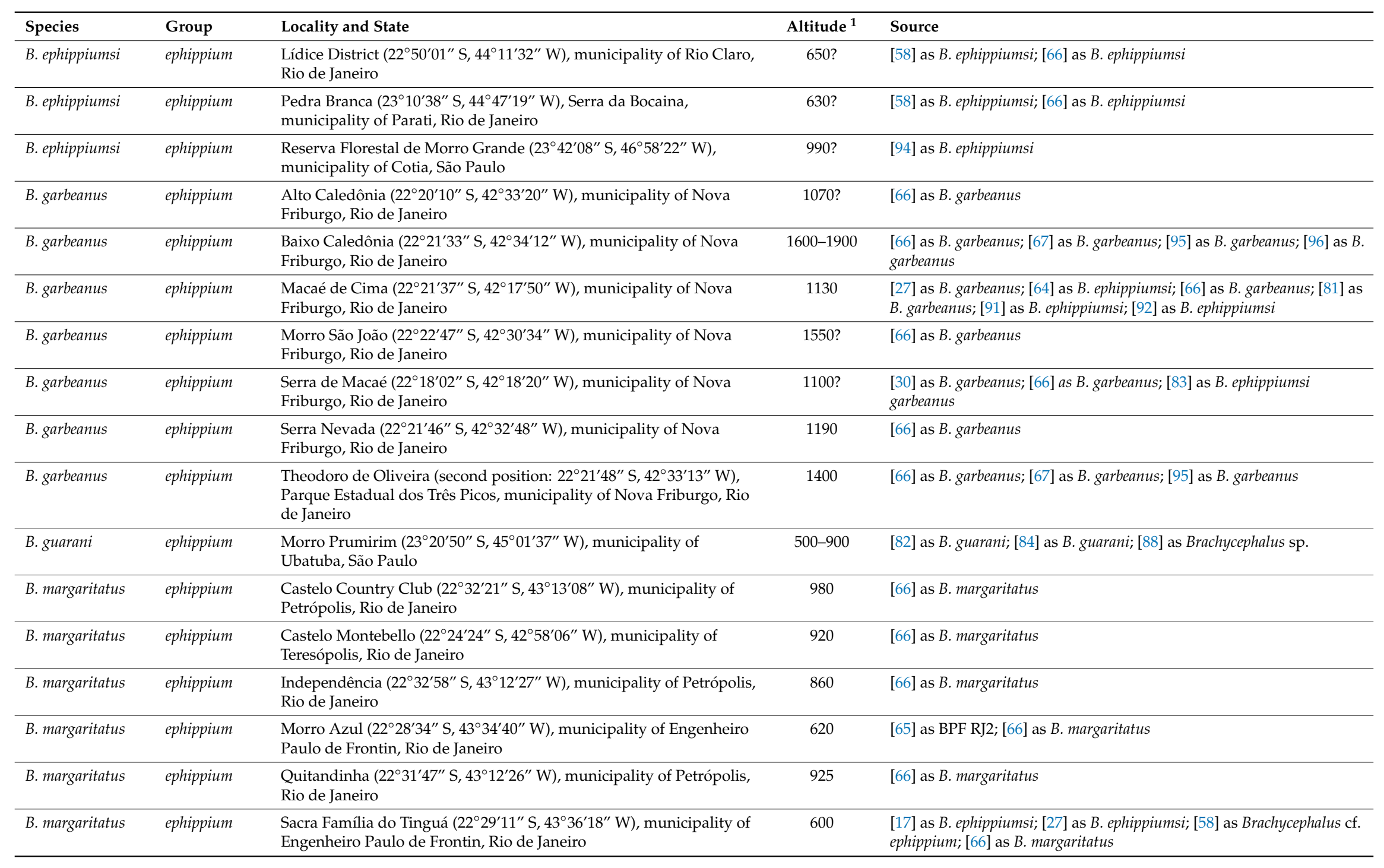


Table 1. Cont

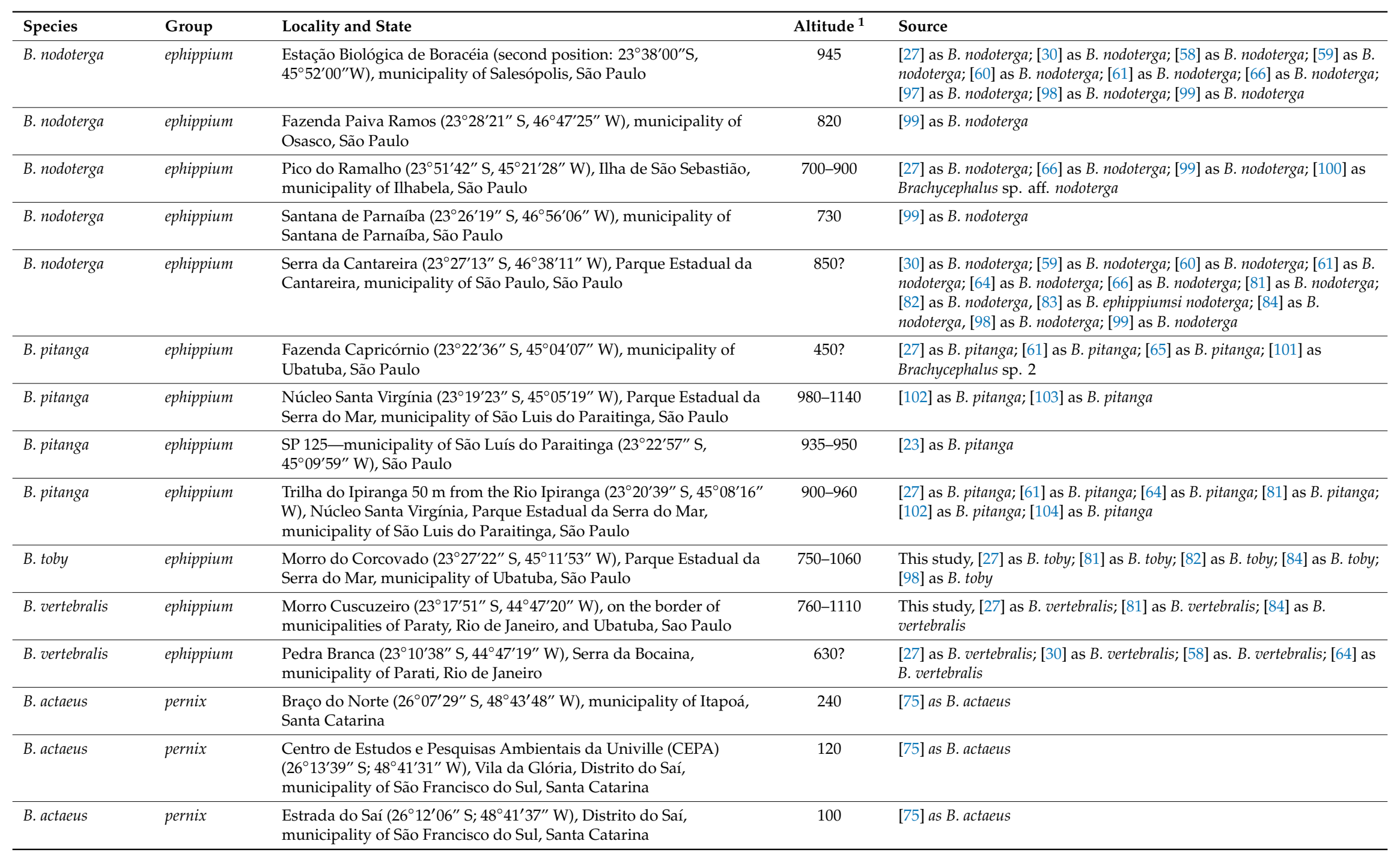


Table 1. Cont

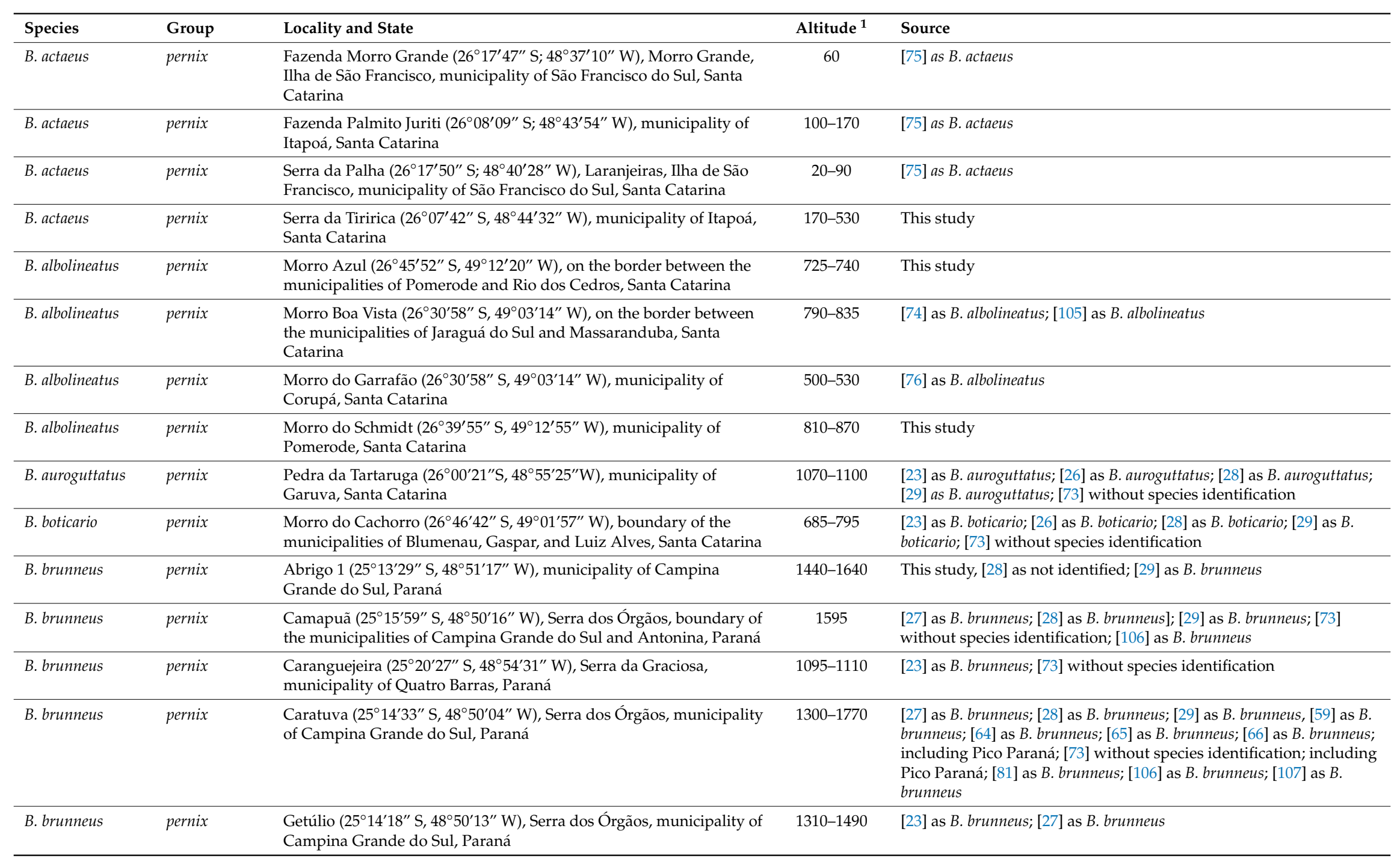


Table 1. Cont

\begin{tabular}{|c|c|c|c|c|}
\hline Species & Group & Locality and State & Altitude $^{1}$ & Source \\
\hline B. brunneus & pernix & $\begin{array}{l}\text { Mãe Catira }\left(25^{\circ} 20^{\prime} 51^{\prime \prime} \text { S, } 48^{\circ} 54^{\prime} 25^{\prime \prime} \text { W), Serra da Graciosa, }\right. \\
\text { municipality of Quatro Barras, Paraná }\end{array}$ & $1135-1405$ & $\begin{array}{l}\text { This study, [27] as Brachycephalus sp. nov. 2; [28] as not identified; } \\
\text { [73] without species identification }\end{array}$ \\
\hline B. coloratus & pernix & $\begin{array}{l}\text { Estância Hidroclimática Recreio da Serra }\left(25^{\circ} 27^{\prime} 14^{\prime \prime} \text { S, } 49^{\circ} 00^{\prime} 27^{\prime \prime} \text { W), }\right. \\
\text { Serra da Baitaca, municipality of Piraquara, Paraná }\end{array}$ & $1145-1230$ & [50] as B. coloratus \\
\hline B. curupira & pernix & $\begin{array}{l}\text { Morro do Canal }\left(25^{\circ} 30^{\prime} 55^{\prime \prime} \text { S, } 48^{\circ} 58^{\prime} 56^{\prime \prime} \text { W), municipality of }\right. \\
\text { Piraquara, Paraná }\end{array}$ & 1320 & $\begin{array}{l}\text { This study, [23] as Brachycephalus sp. 4; [28] as not identified]; [73] } \\
\text { without species identification }\end{array}$ \\
\hline B. curupira & pernix & $\begin{array}{l}\text { Morro do Vigia }\left(25^{\circ} 30^{\prime} 33^{\prime \prime} \mathrm{S}, 48^{\circ} 58^{\prime} 58^{\prime \prime} \mathrm{W}\right) \text {, municipality of Piraquara, } \\
\text { Paraná }\end{array}$ & 1250 & $\begin{array}{l}\text { [23] as Brachycephalus sp. 4; [27] as Brachycephalus sp. nov. 3; [28] as } \\
\text { not identified; [29] as B. curupira; [73] without species identification }\end{array}$ \\
\hline B. ferruginus & pernix & $\begin{array}{l}\text { Olimpo }\left(25^{\circ} 27^{\prime} 03^{\prime \prime} \mathrm{S}, 48^{\circ} 54^{\prime} 59^{\prime \prime} \mathrm{W}\right) \text {, Serra do Marumbi, municipality } \\
\text { of Morretes, Paraná }\end{array}$ & $965-1470$ & $\begin{array}{l}\text { [27] as B. ferruginus; [28] as B. ferruginus; [29] as B. ferruginus, [60] as B. } \\
\text { ferruginus; [64] as B. ferruginus; [66] as B. ferruginus; [73] without } \\
\text { species identification; [81] as B. ferruginus }\end{array}$ \\
\hline B. fuscolineatus & pernix & $\begin{array}{l}\text { Morro Braço da Onça }\left(26^{\circ} 44^{\prime} 58^{\prime \prime} \mathrm{S}, 48^{\circ} 55^{\prime} 41^{\prime \prime} \mathrm{W}\right) \text {, municipality of } \\
\text { Luiz Alves, Santa Catarina }\end{array}$ & $525-530$ & [24] as B. fuscolineatus \\
\hline B. fuscolineatus & pernix & $\begin{array}{l}\text { Morro do Baú }\left(26^{\circ} 47^{\prime} 58^{\prime \prime} \text { S, } 48^{\circ} 55^{\prime} 47^{\prime \prime} \text { W), municipality of Ilhota, }\right. \\
\text { Santa Catarina }\end{array}$ & $640-790$ & $\begin{array}{l}\text { [26] as B. fuscolineatus; [27] as Brachycephalus sp. nov. 9; [28] as B. } \\
\text { fuscolineatus; [29] as B. fuscolineatus; [73] without species identification }\end{array}$ \\
\hline B. leopardus & pernix & $\begin{array}{l}\text { Morro dos Perdidos }\left(25^{\circ} 53^{\prime} 22^{\prime \prime} \mathrm{S}, 48^{\circ} 57^{\prime} 22^{\prime \prime} \mathrm{W}\right) \text {, municipality of } \\
\text { Guaratuba, Paraná }\end{array}$ & $1340-1420$ & $\begin{array}{l}\text { [26] as B. leopardus; [27] as Brachycephalus sp. nov. } 4 ;[28] \text { as B. } \\
\text { leopardus; [73] without species identification }\end{array}$ \\
\hline B. leopardus & pernix & $\begin{array}{l}\text { Serra do Araçatuba }\left(25^{\circ} 54^{\prime} 07^{\prime \prime} \mathrm{S}, 48^{\circ} 59^{\prime} 47^{\prime \prime} \mathrm{W}\right) \text {, municipality of } \\
\text { Tijucas do Sul, Paraná }\end{array}$ & $1640-1645$ & $\begin{array}{l}\text { [26] as B. leopardus; [27] as Brachycephalus sp. nov. 4; [28] as B. } \\
\text { leopardus; [73] without species identification }\end{array}$ \\
\hline B. mariaeterezae & pernix & $\begin{array}{l}\text { Reserva Particular do Patrimônio Natural Caetezal, top of the Serra } \\
\text { Queimada }\left(26^{\circ} 06^{\prime} 51^{\prime \prime} \text { S, } 49^{\circ} 03^{\prime} 45^{\prime \prime} \text { W), municipality of Joinville, Santa }\right. \\
\text { Catarina }\end{array}$ & $1265-1270$ & $\begin{array}{l}\text { [26] as B. mariaeterezae; [27] as Brachycephalus sp. nov. 6; [28] as B. } \\
\text { mariaeterezae; [29] as B. mariaeterezae; [73] without species } \\
\text { identification }\end{array}$ \\
\hline B. mirissimus & pernix & $\begin{array}{l}\text { Morro Santo Anjo }\left(26^{\circ} 37^{\prime} 41^{\prime \prime} \mathrm{S}, 48^{\circ} 55^{\prime} 50^{\prime \prime} \mathrm{W}\right) \text {, municipality of } \\
\text { Massaranduba, Santa Catarina }\end{array}$ & $470-540$ & [25] as B. mirissimus \\
\hline B. olivaceus & pernix & $\begin{array}{l}\text { Base of the Serra Queimada }\left(26^{\circ} 04^{\prime} 57^{\prime \prime} \mathrm{S}, 49^{\circ} 03^{\prime} 59^{\prime \prime} \mathrm{W}\right) \text {, municipality } \\
\text { of Joinville, Santa Catarina }\end{array}$ & 985 & [17] as Brachycephalus sp. nov. 7; [26] as B. olivaceus \\
\hline B. olivaceus & pernix & $\begin{array}{l}\text { Castelo dos Bugres (second position: } 26^{\circ} 13^{\prime} 59^{\prime \prime} \mathrm{S}, 49^{\circ} 03^{\prime} 13^{\prime \prime} \mathrm{W} \text { ), } \\
\text { municipality of Joinville, Santa Catarina }\end{array}$ & $800-835$ & $\begin{array}{l}\text { [26] as B. olivaceus; [27] as Brachycephalus sp. nov. 7; [28] as B. } \\
\text { olivaceus; [73] without species identification; [108] as B. olivaceus }\end{array}$ \\
\hline B. olivaceus & pernix & $\begin{array}{l}\text { Morro do Boi }\left(26^{\circ} 24^{\prime} 42^{\prime \prime} S, 49^{\circ} 12^{\prime} 59^{\prime \prime} \mathrm{W}\right) \text {, municipality of Corupá, } \\
\text { Santa Catarina }\end{array}$ & $650-920$ & [23] as B. olivaceus; [27] as Brachycephalus sp. 3; [29] as B. olivaceus \\
\hline
\end{tabular}


Table 1. Cont

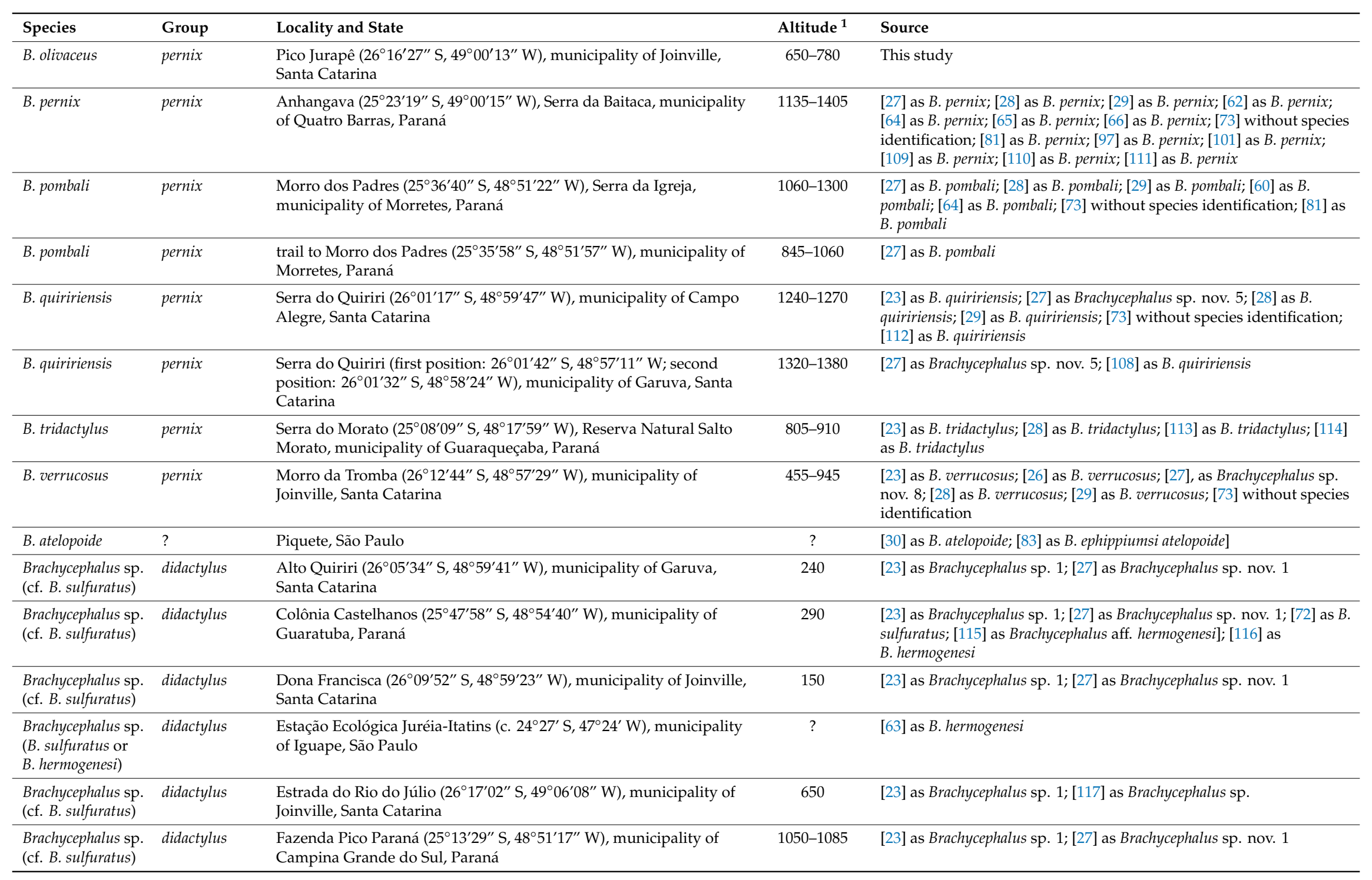


Table 1. Cont

\begin{tabular}{|c|c|c|c|c|}
\hline Species & Group & Locality and State & Altitude $^{1}$ & Source \\
\hline $\begin{array}{l}\text { Brachycephalus sp. } \\
\text { (cf. B. sulfuratus) }\end{array}$ & didactylus & $\begin{array}{l}\text { Fazenda Primavera }\left(24^{\circ} 53^{\prime} 08^{\prime \prime} S, 48^{\circ} 45^{\prime} 51^{\prime \prime} \mathrm{W}\right) \text {, municipality of Tunas } \\
\text { do Paraná, Paraná }\end{array}$ & 1060 & [23] as Brachycephalus sp. 1; [27] as Brachycephalus sp. nov. 1 \\
\hline $\begin{array}{l}\text { Brachycephalus sp. } \\
\text { (B. sulfuratus or } \\
\text { B. hermogenesi) }\end{array}$ & didactylus & Municipality of Ibiúna (c. $23^{\circ} 39^{\prime} \mathrm{S}, 47^{\circ} 13^{\prime}$ W), São Paulo & $?$ & [72] as B. hermogenesi \\
\hline $\begin{array}{l}\text { Brachycephalus sp. } \\
\text { (B. sulfuratus or } \\
\text { B. hermogenesi) }\end{array}$ & didactylus & Municipality of Juquitiba (c. $23^{\circ} 56^{\prime} \mathrm{S}, 47^{\circ} 04^{\prime}$ W), São Paulo & $?$ & [63] as B. hermogenesi; [72] as B. hermogenesi \\
\hline $\begin{array}{l}\text { Brachycephalus sp. } \\
\text { (cf. } \\
\text { B. hermogenesi) }\end{array}$ & didactylus & Municipality of Paraty (c. $23^{\circ} 13^{\prime} 07^{\prime \prime}$ S, $44^{\circ} 43^{\prime} 15^{\prime \prime}$ W), Rio de Janeiro & ? & [18] as B. hermogenesi \\
\hline $\begin{array}{l}\text { Brachycephalus sp. } \\
\text { (B. sulfuratus or } \\
\text { B. hermogenesi) }\end{array}$ & didactylus & Municipality of Peruíbe $\left(24^{\circ} 18^{\prime}\right.$ S, $46^{\circ} 59^{\prime}$ W), São Paulo & ? & [72] as B. hermogenesi \\
\hline $\begin{array}{l}\text { Brachycephalus sp. } \\
\text { (B. sulfuratus or } \\
\text { B. hermogenesi) }\end{array}$ & didactylus & Municipality of Piedade (c. $23^{\circ} 54^{\prime} \mathrm{S}, 47^{\circ} 25^{\prime}$ W), São Paulo & ? & [81] as B. hermogenesi; [118] as B. hermogenesi \\
\hline $\begin{array}{l}\text { Brachycephalus sp. } \\
\text { (B. sulfuratus or } \\
\text { B. hermogenesi) }\end{array}$ & didactylus & Municipality of Registro (c. $24^{\circ} 30^{\prime}$ S, $47^{\circ} 51^{\prime}$ W), São Paulo & $?$ & [72] as B. hermogenesi \\
\hline $\begin{array}{l}\text { Brachycephalus sp. } \\
\text { (B. sulfuratus or } \\
\text { B. hermogenesi) }\end{array}$ & didactylus & Municipality of Ribeirão Grande (c. $24^{\circ} 06^{\prime} \mathrm{S}, 48^{\circ} 22^{\prime}$ W), São Paulo & ? & [63] as B. hermogenesi \\
\hline $\begin{array}{l}\text { Brachycephalus sp. } \\
\text { (B. sulfuratus or } \\
\text { B. hermogenesi) }\end{array}$ & didactylus & Municipality of Tapiraí (c. $23^{\circ} 57^{\prime} 55^{\prime \prime}$ S, $47^{\circ} 30^{\prime} 19^{\prime \prime}$ W), São Paulo & 870 & [63] as B. hermogenesi; [118] as B. hermogenesi \\
\hline $\begin{array}{l}\text { Brachycephalus sp. } \\
\text { (B. sulfuratus or } \\
\text { B. hermogenesi) }\end{array}$ & didactylus & $\begin{array}{l}\text { Parque Estadual de Jacupiranga (c. } 24^{\circ} 38^{\prime} \mathrm{S}, 48^{\circ} 24^{\prime} \mathrm{W} \text { ), municipality } \\
\text { of Eldorado, São Paulo }\end{array}$ & $?$ & [72] as B. hermogenesi \\
\hline $\begin{array}{l}\text { Brachycephalus sp. } \\
\text { (B. hermogenesi or } \\
\text { B. sulfuratus) }\end{array}$ & didactylus & $\begin{array}{l}\text { Parque Natural Municipal Nascentes de Paranapiacaba }\left(23^{\circ} 46^{\prime} 10^{\prime \prime} \text { S, }\right. \\
\left.46^{\circ} 17^{\prime} 36^{\prime \prime} \mathrm{W}\right) \text {, municipality of Santo André, São Paulo }\end{array}$ & 840 & [119] as B. hermogenesi \\
\hline $\begin{array}{l}\text { Brachycephalus sp. } \\
\text { (cf. B. sulfuratus) }\end{array}$ & didactylus & $\begin{array}{l}\text { Pico Agudinho }\left(25^{\circ} 36^{\prime} 24^{\prime \prime} \text { S, } 48^{\circ} 43^{\prime} 33^{\prime \prime} \text { W), Serra da Prata, }\right. \\
\text { municipality of Morretes, Paraná }\end{array}$ & 385 & [23] as Brachycephalus sp. 1; [27] as Brachycephalus sp. nov. 1 \\
\hline
\end{tabular}


Table 1. Cont.

\begin{tabular}{|c|c|c|c|c|}
\hline Species & Group & Locality and State & Altitude $^{1}$ & Source \\
\hline $\begin{array}{l}\text { Brachycephalus sp. } \\
\text { (B. sulfuratus or } \\
\text { B. hermogenesi) }\end{array}$ & didactylus & $\begin{array}{l}\text { Reserva Betary }\left(24^{\circ} 33^{\prime} 08^{\prime \prime} \text { S, } 48^{\circ} 40^{\prime} 49^{\prime \prime} \text { W), municipality of Iporanga, }\right. \\
\text { São Paulo }\end{array}$ & 190 & This study \\
\hline $\begin{array}{l}\text { Brachycephalus sp. } \\
\text { (B. hermogenesi or } \\
\text { B. sulfuratus) }\end{array}$ & didactylus & $\begin{array}{l}\text { Reserva Biológica do Alto da Serra de Paranapiacaba }\left(23^{\circ} 46^{\prime} 40^{\prime \prime} \text { S, }\right. \\
46^{\circ} 18^{\prime} 45^{\prime \prime} \text { W), municipality of Santo André, São Paulo }\end{array}$ & $800-850$ & [23] as B. hermogenesi; [63] as B. hermogenesi; [119] as B. hermogenesi \\
\hline $\begin{array}{l}\text { Brachycephalus sp. } \\
\text { (B. sulfuratus or } \\
\text { B. hermogenesi) }\end{array}$ & didactylus & $\begin{array}{l}\text { Reserva Florestal de Morro Grande }\left(23^{\circ} 42^{\prime} 08^{\prime \prime} \text { S, } 46^{\circ} 58^{\prime} 22^{\prime \prime} \text { W), }\right. \\
\text { municipality of Cotia, São Paulo }\end{array}$ & $990 ?$ & $\begin{array}{l}\text { [23] as B. hermogenesi, [63] as B. hermogenesi; [72] as B. hermogenesi; } \\
\text { [94] as B. hermogenesi }\end{array}$ \\
\hline $\begin{array}{l}\text { Brachycephalus sp. } \\
\text { (cf. B. sulfuratus) }\end{array}$ & didactylus & $\begin{array}{l}\text { Sítio Ananias }\left(25^{\circ} 47^{\prime} 08^{\prime \prime} \mathrm{S}, 48^{\circ} 43^{\prime} 03^{\prime \prime} \mathrm{W}\right) \text {, municipality of Guaratuba, } \\
\text { Paraná }\end{array}$ & 25 & [23] as Brachycephalus sp. 1; [27] as Brachycephalus sp. nov. 1 \\
\hline $\begin{array}{l}\text { Brachycephalus } \\
\text { sp. }\end{array}$ & ephippium & $\begin{array}{l}\text { Paranapiacaba }\left(23^{\circ} 46^{\prime} 30^{\prime \prime} \mathrm{S}, 46^{\circ} 17^{\prime} 57^{\prime \prime} \mathrm{W}\right) \text {, municipality of Santo } \\
\text { André, São Paulo }\end{array}$ & 825 & [27] as Brachycephalus sp. 1; [66] as B. ephippiumsi \\
\hline $\begin{array}{l}\text { Brachycephalus } \\
\text { sp. }\end{array}$ & ephippium & $\begin{array}{l}\text { Parque Natural Municipal Nascentes de Paranapiacaba }\left(23^{\circ} 46^{\prime} 10^{\prime \prime} \mathrm{S} \text {, }\right. \\
46^{\circ} 17^{\prime} 36^{\prime \prime} \text { W), municipality of Santo André, São Paulo }\end{array}$ & $800-1164 ?$ & [120] as Brachycephalus sp. \\
\hline $\begin{array}{l}\text { Brachycephalus } \\
\text { sp. }\end{array}$ & ephippium & $\begin{array}{l}\text { Penísula do Bororé }\left(23^{\circ} 47^{\prime} 11^{\prime \prime} \text { S, } 46^{\circ} 38^{\prime} 45^{\prime \prime} \text { W), Represa Billings, }\right. \\
\text { Grajaú District, municipality of São Paulo, São Paulo }\end{array}$ & 780 & $\begin{array}{l}\text { [27] as Brachycephalus nodoterga; [99] as another species than } B \text {. } \\
\text { nodoterga of [27] }\end{array}$ \\
\hline $\begin{array}{l}\text { Brachycephalus } \\
\text { sp. }\end{array}$ & ephippium & $\begin{array}{l}\text { Reserva Biológica do Alto da Serra de Paranapiacaba }\left(23^{\circ} 46^{\prime} 40^{\prime \prime} \text { S, }\right. \\
46^{\circ} 18^{\prime} 45^{\prime \prime} \text { W), municipality of Santo André, São Paulo }\end{array}$ & 800 & [27] as Brachycephalus sp. 1 \\
\hline $\begin{array}{l}\text { Brachycephalus } \\
\text { sp. }\end{array}$ & ephippium & $\begin{array}{l}\text { Theodoro de Oliveira (first position: } 22^{\circ} 22^{\prime} 11^{\prime \prime} \mathrm{S}, 42^{\circ} 33^{\prime} 25^{\prime \prime} \mathrm{W} \text { ), } \\
\text { Parque Estadual dos Três Picos, municipality of Nova Friburgo, Rio } \\
\text { de Janeiro }\end{array}$ & $1100-1200$ & [67] as Brachycephalus sp.; [95] as Brachycephalus sp. nov. \\
\hline $\begin{array}{l}\text { Brachycephalus } \\
\text { sp. }\end{array}$ & pernix & $\begin{array}{l}\text { Pedra Branca do Araraquara }\left(25^{\circ} 56^{\prime} 00^{\prime \prime} \mathrm{S}, 48^{\circ} 52^{\prime} 50^{\prime \prime} \mathrm{W}\right) \text {, Serra do } \\
\text { Araraquara, municipality of Guaratuba, Paraná }\end{array}$ & 1000 & [23] as Brachycephalus sp. 5 \\
\hline $\begin{array}{l}\text { Brachycephalus } \\
\text { sp. }\end{array}$ & pernix & $\begin{array}{l}\text { Pico Paraná }\left(25^{\circ} 15^{\prime} 10^{\prime \prime} \mathrm{S}, 48^{\circ} 48^{\prime} 32^{\prime \prime} \mathrm{W}\right) \text {, Serra dos Órgãos, } \\
\text { municipality of Antonina, Paraná }\end{array}$ & 1880 & This study \\
\hline $\begin{array}{l}\text { Brachycephalus } \\
\text { sp. }\end{array}$ & pernix & $\begin{array}{l}\text { Serra Canasvieiras }\left(25^{\circ} 36^{\prime} 58^{\prime \prime} \mathrm{S}, 48^{\circ} 46^{\prime} 59^{\prime \prime} \text { W), boundary of the }\right. \\
\text { municipalities of Guaratuba and Morretes, Paraná }\end{array}$ & 1080 & $\begin{array}{l}\text { [23] as Brachycephalus sp. 5; [25] as B. sp. Canasvieiras; [28] as not } \\
\text { identified; [73] without species identification }\end{array}$ \\
\hline $\begin{array}{l}\text { Brachycephalus } \\
\text { sp. }\end{array}$ & pernix & $\begin{array}{l}\text { Tupipiá ( } 25^{\circ} 15^{\prime} 13^{\prime \prime} \text { S, } 48^{\circ} 48^{\prime} 20^{\prime \prime} \text { W), Serra dos Órgãos, municipality of } \\
\text { Antonina, Paraná }\end{array}$ & 1560 & $\begin{array}{l}\text { This study, [27] as B. brunneus; [28] as B. brunneus; [29] as B. sp. } \\
\text { Tupipiá, [73] without species identification }\end{array}$ \\
\hline $\begin{array}{l}\text { Brachycephalus sp. } \\
\text { (cf. B. darkside } \\
\text { juvenile) }\end{array}$ & $?$ & $\begin{array}{l}\text { Parque Estadual da Serra do Brigadeiro (cf. } 20^{\circ} 43^{\prime} 16^{\prime \prime} \mathrm{S}, 42^{\circ} 29^{\prime} 05^{\prime \prime} \\
\text { W), municipality of Araponga, Minas Gerais }\end{array}$ & $1330 ?$ & [85] as Brachycephalus cf. didactylus \\
\hline
\end{tabular}

${ }^{1}$ Data with "?" were not available in literature. 
The main threat to the species of Brachycephalus is deforestation, affecting not only their EO but also other aspects of their biology, such as population size and individual health. Indeed, deforestation affects 20 species. Other species are under threat due to their small EO. Forests within EOs were converted into agricultural areas (e.g., for coffee and palm plantations - Archontophoenix alexandrae H. Wendl. \& Drude), tree monocultures (Pinus spp. and Eucalyptus spp.), urban areas and, more frequently, pastures. Some species also have part of their EO flooded by dams (e.g., B. nodoterga) or affected by landslides (e.g., B. izecksohni). Fire, edge effects, timber harvest, grazing, and the invasion of exotic plants are impacts that reduce the quality of EO. For instance, deforestation and loss of habitat quality are important threats to B. mariaeterezae, whose type locality suffers from fire, grazing, and timber harvests. Fire and grazing substantially affect the quality of forests, even the cloud forests of B. quiririensis. The estimated population sizes were above those used in the IUCN criteria and therefore were not useful to rank the studied species regarding their conservation status.

The conservation status of all described species (Figure 2, Table 2) were determined. Twenty-one species (58.3\% of all species) were classified as at risk of extinction: six as CR $(28.6 \%)$, five as Endangered (EN; $23.8 \%$ ), and 10 as Vulnerable (VU; $47.6 \%$ ). Five species (13.9\% of all species) were classified as non-threatened $(=\mathrm{LC})$ and the remaining ten species $(27.8 \%)$ as DD. The reduced EO (criteria B1) contributes to the ranking status of the conservation of 16 species associated with the number of locations (criteria B1a; 16 species), threats that reduce the area of EO (criteria B1b(i); 16 species), and quality of the area of EO (B1b(iii); 16 species; Table 2). B2 criteria were adopted for eight species (Table 2). For the B2 criteria, the number of locations (criteria B2a; eight species) and the threats that reduced the $\mathrm{AO}$ area (criteria $\mathrm{B} 2 \mathrm{~b}$ (ii); eight species) and quality (criteria $\mathrm{B} 2 \mathrm{~b}(\mathrm{iii})$; eight species) were also considered. Only one additional criterion (D2) for five species with less than 2000 ha of AO was used, and no threat could be assessed for this AO.

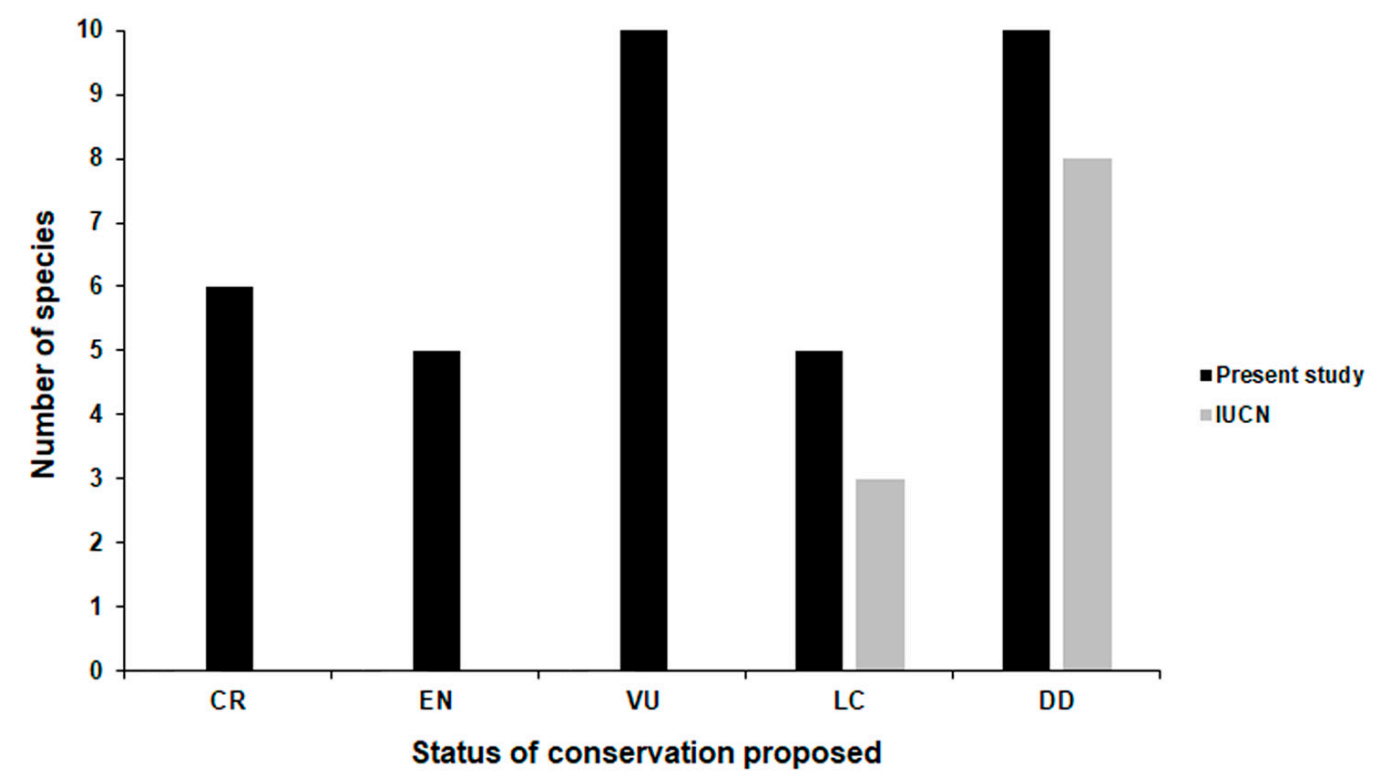

Figure 2. Status of conservation for the 36 species of Brachycephalus proposed in this study according to the IUCN [48] criteria and categories proposed by IUCN [31-41]. Abbreviation: CR = Critically Endangered; $\mathrm{EN}=$ Endangered; $\mathrm{VU}=$ Vulnerable; $\mathrm{LC}=$ Least Concern; DD = Data Deficient. 
Table 2. Status of conservation of identified species of Brachycephalus according to IUCN [48]. Abbreviations: EO = extent of occurrence (see text for details); MMA = Ministério do Meio Ambiente (Brazil).

\begin{tabular}{|c|c|c|c|c|c|c|c|c|c|c|c|}
\hline \multirow{2}{*}{ Species $^{1}$} & \multirow{2}{*}{ Localities $^{1}$} & \multirow{2}{*}{$\begin{array}{l}\text { Altitudinal Range } \\
\text { (m a.s.l.) }{ }^{1,2}\end{array}$} & \multicolumn{2}{|c|}{ Evaluation of EO (ha) } & \multirow{2}{*}{$\begin{array}{l}\text { Flow Chart } \\
\text { Pathway }^{3}\end{array}$} & \multicolumn{2}{|c|}{ Population } & \multicolumn{4}{|c|}{ Conservation Status-Criteria } \\
\hline & & & Previous $^{2}$ & This Study ${ }^{2}$ & & Locations $^{2}$ & Individuals ${ }^{2,3}$ & IUCN & MMA & Others & This Study \\
\hline \multicolumn{12}{|c|}{ B. didactylus group } \\
\hline B. didactylus & 8 & $35-1110$ & - & $702,983.4$ & 5 & 4 & $79,655,049$ & LC [41] & - & - & VU - B1ab(i,iii) \\
\hline B. hermogenesi & 11 & $0-1090$ & $567,589.9[23]$ & $143,325.0$ & 5 & 1 & $?$ & LC [33] & - & - & VU - B $1 \mathrm{ab}(\mathrm{i}, \mathrm{iii})$ \\
\hline B. pulex & 1 & $800-930$ & $488.2[23]$ & 482.3 & 1 & 1 & $?$ & - & - & - & VU - D2 \\
\hline B. sulfuratus & 26 & $40-1205$ & $778,458.4[23]$ & $3,021,786.1$ & 5 & 1 & $302,178,610$ & - & - & - & LC \\
\hline \multicolumn{12}{|c|}{ B. ephippiumsi group } \\
\hline B. alipioi & 3 & $1070-1100$ & $\begin{array}{l}38,950.0[47], \\
27,930.0[43]\end{array}$ & $1,706.1$ & 3 & 1 & $?$ & DD [35] & NT [43] & - & CR - B1ab(i,iii) \\
\hline B. bufonoides & 1 & $?$ & - & ? & 4 & ? & ? & - & - & - & DD \\
\hline B. crispus & 1 & $800-1190$ & ? & $?$ & 2 & 1 & ? & - & - & - & DD \\
\hline B. darkside & 2 & $1265-1500$ & - & $5,700.8$ & 1 & 1 & $?$ & - & - & - & CR - B1ab(i,iii) \\
\hline B. ephippiumsi & 31 & $200-1250$ & ? & $1,792,535.1$ & 5 & 6 & $13,336,461$ & LC [38] & - & - & VU - B1ab(i,iii) \\
\hline B. garbeanus & 7 & 1130-1900 & $12,268.0[23]$ & $6,426.5$ & 5 & 2 & $?$ & - & - & - & $\begin{array}{l}\text { EN - } \\
\text { B1ab(i,iii)+2ab(ii,iii) }\end{array}$ \\
\hline B. guarani & 1 & $500-900$ & $?$ & $?$ & 2 & 1 & ? & - & - & - & DD \\
\hline B. margaritatus & 6 & $600-980$ & $18,272.9[23]$ & $10,710.5$ & 5 & 2 & ? & - & - & - & EN - B1ab(i,iii) \\
\hline B. nodoterga & 5 & $700-900$ & $\begin{array}{r}9,690.0[47], \\
108,280.0[42]\end{array}$ & 28.458 .1 & 5 & 3 & ? & DD [31] & $\mathrm{DD}[42]$ & - & VU - B1ab(i,iii) \\
\hline B. pitanga & 4 & $900-1140$ & $2,377.1[23]$ & $2,245.1$ & 5 & 1 & $29,157,136$ & - & - & - & LC \\
\hline B. toby & 1 & $750-1060$ & $?$ & $?$ & 2 & 1 & $?$ & - & - & - & DD \\
\hline B. vertebralis & 2 & $760-1110$ & $\begin{array}{c}161,990.0[47], \\
18,580.0[44]\end{array}$ & $?$ & 2 & 2 & ? & DD [37] & DD [44] & - & $\mathrm{DD}$ \\
\hline \multicolumn{12}{|c|}{ B. pernix group } \\
\hline B. actaeus & 7 & $20-530$ & - & $15,841.6$ & 6 & 2 & $?$ & - & - & - & $\begin{array}{c}\text { EN - } \\
\text { B1ab(i,iii)+2ab(ii,iii) }\end{array}$ \\
\hline B. albolineatus & 4 & 500-835 & $34.4[74]$ & $2,784.4$ & 5 & 12 & $1,076,087$ & - & - & DD [74] & VU - B1ab(i,iii) \\
\hline B. auroguttatus & 1 & $1070-1100$ & - & $?$ & 3 & 1 & & - & - & - & DD \\
\hline B. boticario & 1 & $685-795$ & $11.1[23]$ & 38.8 & 1 & 1 & $?$ & - & - & - & $\begin{array}{c}\text { CR - } \\
\text { B1ab(i,iii)+2ab(ii,iii) }\end{array}$ \\
\hline B. brunneus & 6 & $1095-1770$ & $\begin{array}{c}1,100.0[47], 5,687.1 \\
{[23]}\end{array}$ & $5,385.6$ & 6 & 2 & $?$ & DD [39] & - & - & LC \\
\hline B. coloratus & 1 & $1145-1230$ & - & 37.4 & 1 & 1 & & - & - & DD [50] & VU - D2 \\
\hline B. curupira & 3 & $1095-1320$ & $2,211.54[23]$ & $4,751.4$ & 6 & 2 & $21,117,312$ & - & - & DD [50] & LC \\
\hline B. ferruginus & 1 & $965-1,470$ & $\begin{array}{c}38,950.0[47], \\
5,475.5[23]\end{array}$ & $5,994.3$ & 1 & 1 & $?$ & DD [34] & - & - & LC \\
\hline B. fuscolineatus & 2 & $525-790$ & $23.63[23], 23.8$ [24] & 23.8 & 1 & 2 & 119,000 & - & - & - & $\begin{array}{c}\text { CR - } \\
\text { B1ab(i,iii)+2ab(ii,iii) }\end{array}$ \\
\hline B. izecksohni & 1 & $980-1340$ & $\begin{array}{c}1,100.0[47], 350.4 \\
{[23]}\end{array}$ & 378.3 & 1 & 1 & $?$ & DD [40] & - & - & VU - D2 \\
\hline B. leopardus & 2 & $1340-1645$ & $176.7[23]$ & 363.1 & 1 & 3 & $?$ & - & - & - & $\begin{array}{c}\text { EN - } \\
\text { B1ab(i,iii)+2ab(ii,iii) }\end{array}$ \\
\hline
\end{tabular}


Table 2. Cont

\begin{tabular}{|c|c|c|c|c|c|c|c|c|c|c|c|}
\hline \multirow{2}{*}{ Species $^{1}$} & \multirow{2}{*}{ Localities $^{1}$} & \multirow{2}{*}{$\begin{array}{l}\text { Altitudinal Range } \\
\text { (m a.s.l.) })^{1,2}\end{array}$} & \multicolumn{2}{|c|}{ Evaluation of EO (ha) } & \multirow{2}{*}{$\begin{array}{l}\text { Flow Chart } \\
\text { Pathway }^{3}\end{array}$} & \multicolumn{2}{|c|}{ Population } & \multicolumn{4}{|c|}{ Conservation Status_Criteria } \\
\hline & & & Previous $^{2}$ & This Study ${ }^{2}$ & & Locations $^{2}$ & Individuals 2,3 & IUCN & MMA & Others & This Study \\
\hline B. mariaeterezae & 1 & $1265-1270$ & - & ? & 3 & 1 & $?$ & - & - & - & DD \\
\hline B. mirissimus & 1 & $470-540$ & $56.8[25]$ & 56.8 & 1 & 1 & 78,344 & - & - & CR [25] & $\begin{array}{c}\text { CR - } \\
\text { B1ab(i,iii)+2ab(ii,iii) }\end{array}$ \\
\hline B. olivaceus & 4 & $650-985$ & $12,531.6[23]$ & $18,850.1$ & 5 & 2 & $?$ & - & - & - & $\begin{array}{c}\text { EN - } \\
\text { B1ab(i,iii)+2ab(ii,iii) }\end{array}$ \\
\hline B. pernix & 1 & 1135-1405 & $\begin{array}{c}1,950.0[47], 432.1 \\
{[23], 400[45]}\end{array}$ & 389.4 & 1 & 1 & $?$ & $\mathrm{DD}[32]$ & $\begin{array}{c}\text { CR - } \\
\text { B1ab(iii)+2ab(iii) } \\
\text { [45] }\end{array}$ & - & VU - D2 \\
\hline B. pombali & 2 & $845-1300$ & $31,300.0[47]$ & ? & 2 & 1 & ? & $\mathrm{DD}[36]$ & - & - & DD \\
\hline B.quiririensis & 2 & $1240-1380$ & $1339.0[23]$ & 629.0 & 1 & 1 & ? & - & - & - & $\begin{array}{c}\text { CR - } \\
\text { B1 }\end{array}$ \\
\hline B. tridactylus & 1 & $805-910$ & 41.4 [23] & 41.4 & 1 & 1 & ? & - & - & - & VU - D2 \\
\hline B. verrucosus & 1 & $455-945$ & - & $?$ & 2 & 1 & ? & - & - & - & DD \\
\hline \multicolumn{12}{|c|}{ Incertae sedis } \\
\hline B. atelopoide & 1 & $?$ & - & $?$ & 4 & $?$ & $?$ & - & - & - & DD \\
\hline
\end{tabular}

${ }^{1}$ According Table $1 .{ }^{2}$ Data with "?" could not be estimate because of lack of information in literature. ${ }^{3}$ According Figure 1. 


\section{Discussion}

Based on the assessments, the number of endangered species of Brachycephalus should increase from one to 21 (Table 2). This is a significant shift and poses the question regarding why only one species had been formally recognized as threatened until now (Table 2). There are two possible reasons: (1) a delay due to the fact that many species have only been recently described and (2) a resistance based on current policies of the MMA to change a species conservation status in a short period of time (see below). Twenty-two species have been described in the last 10 years, 14 of which are only known from their type locality, and there is a natural tendency to expect them to be more widely distributed; however, studies in recent years have been gradually revealing new species rather than new records of known species, and new records of already described species have not substantially altered their geographical distributions (e.g., [24,29,76,99]). For example, a new locality record for B. fuscolineatus published after its description increased its EO by just 0.19 ha [24], and this species still has the smallest estimated EO for any Brachycephalus species (Table 2). Two new records of B. curupira (Table 1, [29]) double its EO, which remains small (= 4751.4 ha; Table 2). A new record of B. albolineatus published after its description [76] and two new localities included in Table 1 substantially extend its EO, but as in the case of B. curupira, this remains small (= 2784.4 ha; Table 2). A new record for B. nodoterga [99] did not change the EO of the species because it is located within its EO polygon. Brachycephalus was not found in 13 localities from southern São Paulo to northeastern Santa Catarina with an altitude comparable to other localities where Brachycephalus populations were present. Overall, the reduced geographical distributions of Brachycephalus is the rule for the montane species of the genus, i.e., the B. ephippiumsi and B. pernix groups [23]. Brachycephalus ephippium is the only exception of a montane species group with a large EO, but it is expected that some, if not all, populations may be identified as distinct species in future studies [23].

With respect to the resistance to incorporate drastic changes into the official number of endangered Brachycephalus species, this proposal is warranted despite the current policy of the MMA indicated. The MMA joined several international agreements that set targets for the conservation of the country's threatened biodiversity, and these efforts have been implemented in the successful execution of National Action Plans for the Conservation of Brazilian Endangered Species (Planos de Ação Nacional - PANs). The national scientific community and the MMA have been working together to list threats and conservation actions to all threatened species of the country and to review and to monitor these actions annually. This is possibly the reason that the MMA tended to prefer moving forward with conservation strategies of species that are already listed as threatened rather than revising the list. The effort to prioritize conservation initiatives prior to substantial updates to the list of endangered species is recognized, but the need for MMA to revise the list and to recognize the species listed in this article as threatened is also acknowledged given that they are not yet legally protected.

The most prevalent threat to Brachycephalus is deforestation, much of which is no longer done with heavy machinery and chainsaws. Recently, deforestation in the Atlantic Rainforest has become more subtle and involves the selective removal of trees and shrubs, particularly through inconspicuous strategies, such as bark girdling, which leads to the opening of the canopy and an increased tendency for wind to knock down additional trees. These actions are deliberately conducted a few meters into the forest edge to avoid detection by environmental inspectors. This type of deforestation has been carried out at an alarming rate in Paraná and in the northeast of Santa Catarina for at least 25 years to cultivate bananas, and more recently, to cultivate palm trees (Archontophoenix alexandrae). Deforestation for agricultural activities could also result in soil contamination, affecting species that depend on specific microhabitats and that have permeable skin [121]. Finally, deforestation could exacerbate edge effects, altering microhabitats and microclimatic conditions, which changes sunlight exposure, soil moisture, and plant species composition in the edges [15]. Indeed, B. fuscolineatus was not encountered in forest edges but only in more nuclear vegetation [24].

Deforestation in lowlands can lead to a decrease in the altitudinal distribution of cloud forests [122], potentially shifting the distributions of montane species of Brachycephalus to higher altitudes. This 
possibility of altitudinal species displacement could also be driven by climate change [23]. In tropical forests, temperatures can vary from $0.4{ }^{\circ} \mathrm{C}$ to $0.7^{\circ} \mathrm{C}$ per $100 \mathrm{~m}$ altitude variation [123]. The thermal variation in the altitudinal gradient in a site with the occurrence of Brachycephalus (B. pernix) was determined as $0.56{ }^{\circ} \mathrm{C}$ of the reduction every $100 \mathrm{~m}$ of altitude [124]. A difference in the precipitation levels at this site was also evaluated, with an increase of $40 \mathrm{~mm}$ in mean annual precipitation every $100 \mathrm{~m}$ at elevation [124]. Studies on litter anurans of the Atlantic Rainforest, including Brachycephalus, have shown that population densities are particularly affected by air humidity, air temperature, and altitude $[52,55,78,90]$. This climatic dependence and its relationship with the altitude gradient raises concerns for the long-term conservation of Brachycephalus species that occur in mountains with a restricted altitudinal amplitude.

Lowering the category of threat for B. pernix from CR [45] to VU (Table 2) is proposed. The effects of trampling and timber harvests by tourists in the type locality of the species are likely to be minor, which is entirely distributed within a protected area (Parque Estadual Serra da Baitaca; Table S1). There is a threat of fire in part of the EO of the species, but the vegetation cover is regenerating well in this area after many years of management by volunteer mountaineers, reducing fire susceptibility.

It is recognized that there is some level of subjectivity to apply EO and the number of locations of threatened species. This is because each parameter shows some overlap between EN and VU categories. Brachycephalus hermogenesi and B. nodoterga fit the EN category, but both are recommended to be considered for the VU category because part of their EO is in protected areas (Table S1).

The presence of threatened Brachycephalus in protected areas is a useful tool to rank the species for which conservation actions are more urgent. In Table S1, 10 species without records in protected areas are recognized with three classified as CR (B. boticario, B. mirissimus, and B. quiririensis), two as EN (B. actaeus and B. leopardus), one as VU (B. albolineatus), and four as DD (B. atelopoide, B. auroguttatus, B. bufonoides, $B$. leopardus, and $B$. verrucosus). There are no known living populations of two species ( $B$. atelopoide and $B$. bufonoides). The remaining eight species belonging to the $B$. pernix group occur in southern Paraná (B. leopardus) and Santa Catarina (B. actaeus, B. albolineatus, B. auroguttatus, B. boticario, B. mirissimus, B. quiririensis, and B. verrucosus). Also, it is argued that DD species need special attention to direct further studies to complete adequate assessments of their conservation status as soon as possible.

Santa Catarina stands out as the state in which emergency conservation actions should converge. Creating protected areas is an important way to protect species, however, the conservation of the top three priority species would require the creation of three new protected areas. A protected area for the CR B. quiririensis could already house other species of Brachycephalus that are not in any reserve, namely B. leopardus (EN) and B. auroguttatus (DD). Nonetheless, to be effective, a protected area would first require the expropriation of the land in addition to management actions aimed at recovering forest quality. Given that there are dozens of protected areas waiting for expropriation, this path to conservation does not seem likely at the moment. Private protected areas are an alternative (e.g., Reserva Particular do Patrimônio Natural-RPPN), and some of them already protect two species of Brachycephalus (B. mariaeterezae and B. tridactylus; Table 1 and Table S1). This is the most stable category of protected areas in Brazil and cannot be undone; however, one aspect that does not stimulate the creation of more private protected areas is the lack of government incentives to private owners, except for exemption from territorial taxes. There is an impediment to transferring public financial resources to private persons, even if they are addressing conservation measures.

The conservation of Brachycephalus should also include alternatives to the creation of protected areas. One approach would be to lease land with the occurrence of threatened Brachycephalus at a percentage of the regional value of production per hectare of mountainous lands, which would be an incentive for landowners to leave their land intact. This must be governed by a renewable contract. For this strategy to be put into practice, it is vital to attract international resources. It would also be interesting to attract additional resources of the lease value to promote environmental recovery. The management of invasive alien species, both plants and animals, is unfortunately incipient in Brazil due 
to the high involved costs, thus discouraging the proposition of new management projects. The state of Santa Catarina has emphasized its concern with the conservation of microendemic anurans in its region, creating a specific program for this purpose (Portaria Instituto Estadual do Meio Ambiente IMA N ${ }^{\circ} 283 / 2018$ - 19/12/2018). This is an interesting effort that can put actions discussed into practice and can also result in other effective and innovative actions for the conservation of Brachycephalus in Santa Catarina. In the long term, successful practices can be replicated in other regions of Brazil.

\section{Conclusions}

Advances in knowledge regarding the geographical distribution of the Brachycephalus species have confirmed that they are in fact restricted, and this restriction is the reason for classifying $58 \%$ of the species of the genus as threatened according to IUCN criteria. Restricted geographical distributions should be considered an attribute of the species of the Brachycephalus montane groups. This coincides with the tendency of species with small ranges to be geographically concentrated and disproportionately under the threat of extinction [125] as well as with the tendency of newly described species to be more threatened than those described earlier [3]. With an increased understanding of the nature of most Brachycephalus species as microendemic species, international (IUCN) and national (MMA) agencies might be more likely to update their conservation status based on this proposal. Furthermore, Brazil has the highest amphibian richness in the world and the highest description of new species in recent years, but it is one of the countries with the lowest update rates of conservation status [3].

Deforestation and loss of habitat quality impact almost all species of Brachycephalus (22 species). Species of the genus are locally highly abundant, but they respond in density and geographical distribution to temperature and humidity $[23,24,27]$, which vary along the altitudinal gradient $[122,124]$. Climate change can influence climatic conditions along the altitudinal gradient, confining the distribution of species even further to higher altitudes in the future.

The common action to protect endangered species in Brazil is to create protected areas. The creation of a new protected area in southern Paraná (Serra do Araçatuba) and adjacent to Santa Catarina (Serra do Quiriri) is proposed, but only because it would protect three species (B. quiririensis-CR, B. leopardus-EN, and B. auroguttatus-DD). In the marshes and grasslands associated with the forest of occurrence of these three Brachycephalus species is another endangered frog, Melanophryniscus biancae, which is a candidate for EN $[126,127]$. One reserve including the distribution of these four species would have about 11,000 ha $-6,000$ ha of forests, and 5,000 ha of grasslands [126] - and would also protect the springs of important rivers, such as the Negro, Cubatão, and Pirabeiraba. The creation of several other protected areas to safeguard the remaining threatened species without occurrence in reserves is impractical in the current Brazilian economic scenario. A program to lease strategic private land for owners to keep them intact with the support of international resources is a possibility for the conservation of the species in the short and medium term.

Supplementary Materials: The following are available online at http://www.mdpi.com/1424-2818/11/9/150/s1, Table S1: Records and distribution of the extent of occurrence (EO) of the species of Brachycephalus in relation to protected areas (PA).

Author Contributions: Conceptualization, M.R.B.; methodology, M.R.B.; formal analysis, M.R.B.; investigation, M.R.B.; data curation, M.R.B.; writing—original draft preparation, M.R.B. and L.T.; writing—review and editing, M.R.B., L.T. and M.R.P.

Funding: Field researches was partially funded by Fundação Grupo Boticário de Proteção à Natureza (through grants 0895_20111 and A0010_2014, conducted by Mater Natura-Instituto de Estudos Ambientais) and National Geographic Society (through the grant EC-50722R-18 to L.T.) L.T. was supported through a grant from CAPES / Reitoria and M.R.P. through a grant from CNPq/MCT (571334/2008-3).

Acknowledgments: Luiz F. Ribeiro provided valuable assistance during the field work. We thank two anonymous reviewers for comments on the manuscript.

Conflicts of Interest: The authors declare no conflict of interest. The funders had no role in the design of the study, the collection, analyses, or interpretation of data, the writing of the manuscript, or the decision to publish the results. 


\section{References}

1. Frost, D.R. Amphibian Species of the World: An Online Reference, Version 6.0. 2019. Available online: http://research.amnh.org/herpetology/amphibia/index.html (accessed on 22 June 2019).

2. Köhler, K.; Vieites, D.R.; Bonett, R.M.; Garcia, F.H.; Glaw, F.; Steinke, D.; Vences, M. New Amphibians and Global Conservations: A boost in species discoveries in a highly endangered vertebrate group. BioScience 2005, 55, 693-696. [CrossRef]

3. Tapley, B.; Michaels, C.J.; Gumbs, R.; Böhm, M.; Luedtke, J.; Pearce-Kelly, P.; Rowley, J.J.L. The disparity between species description and conservation assessment: A case study in taxa with high rates of species discovery. Biol. Conserv. 2018, 220, 209-214. [CrossRef]

4. Wake, D.B.; Vredenburg, V.T. Are we in the midst of the sixth mass extinction? A view from the world of amphibians. Proc. Natl. Acad. Sci. USA 2008, 105, 11466-11473. [CrossRef] [PubMed]

5. IUCN. The IUCN Red List of Threatened Species. Available online: https://www.iucnredlist.org (accessed on 28 June 2019).

6. Stuart, S.; Chanson, J.; Cox, N. Status and trends of amphibian declines and extinctions worldwide. Science 2004, 306, 1783-1786. [CrossRef] [PubMed]

7. Skerrat, L.F.; Berger, L.; Speare, R.; Cashins, S.; McDonald, K.R. Spread of Chytridiomycosis Has Caused the Rapid Global Decline and Extinction of Frogs. EcoHealth 2007, 4, 126. [CrossRef]

8. Stuart, S.N.; Hoffmann, M.; Chanson, J.S.; Cox, N.A.; Berridge, R.J.; Ramani, P.; Young, B.E. Threatened Amphibians of the World; Lynx Editions: Barcelona, Spain, 2008; pp. 1-151.

9. Lips, K.P.R.; Burrowes, P.A.; Mendelson, J.R., III; Parra-Olea, G. Amphibian declines in Latins America: A synthesis. Biotropica 2005, 37, 222-226. [CrossRef]

10. Ruland, F.; Jeschke, J.M. Threat-dependent traits of endangered frogs. Biol. Conserv. 2017, 206, 310-313. [CrossRef]

11. Ruggeri, J.; Ribeiro, L.P.; Pontes, M.R.; Toffolo, C.; Candido, M.; Carriero, M.M.; Zanella, N.; Sousa, R.L.M.; Toledo, L.F. First case of wild amphibians infected with Ranavirus in Brazil. J. Wildlife Dis. 2019, 55. Preprint.

12. Myers, N.; Mittermeier, R.A.; Mittermeier, C.G.; Fonseca, G.A.B.; Kent, J. Biodiversity hotspots for conservation priorities. Nature 2000, 403, 853-858. [CrossRef] [PubMed]

13. Dov-Por, F. Sooretema, the Atlantic Rain Forest of Brazil; SPB Academic Publishing: The Hague, The Netherlands, 1992; Volume 128.

14. MMA. Mapa de Vegetação Nativa na Área de Aplicação da Lei no. 11.428/2006 —Lei da Mata Atlântica (ano base 2009); Ministério do Meio Ambiente: Brasília, Brazil, 2015; pp. 1-85.

15. Rezende, C.L.; Scarano, F.R.; Assad, E.D.; Joly, C.A.; Metzger, J.P.; Strassburg, B.B.N.; Tabarelli, M.; Fonseca, G.A.; Mittermeier, R.A. From hotspots to hopespot: An opportunity for the Brazilian Atlantic Forest. Perspect. Ecol. Conserv. 2018, 16, 204-214. [CrossRef]

16. Ministério do Meio Ambiente. Livro Vermelho da Fauna Brasileira Ameaçada de Extinção—Sumário Executivo; Instituto Chico Mendes de Conservação da Biodiversidade_ICMBio: Brasília, Brazil, 2016; pp. 1-76.

17. Izecksohn, E. Novo gênero e nova espécie de Brachycephalidae do Estado do Rio de Janeiro, Brasil. Boletim do Museu Nacional Zoologia 1971, 280, 1-12.

18. Giaretta, A.A.; Sawaya, R.J. Second species of Psyllophryne (Anura: Brachycephalidae). Copeia 1998, 1998, 985-987. [CrossRef]

19. Pombal, J.P., Jr. Oviposição e desenvolvimento de Brachycephalus ephippium (Spix) (Anura, Brachycephalidae). Rev. Brasil. Zool. 1999, 16, 967-976. [CrossRef]

20. Napoli, M.F.; Caramaschi, U.; Cruz, C.A.G.; Dias, I.R. A new species of flea-toad, genus Brachycephalus Fitzinger (Amphibia: Anura: Brachycephalidae), from the Atlantic Rainforest of southern Bahia, Brazil. Zootaxa 2011, 2739, 33-40. [CrossRef]

21. Yeh, J. The effect of miniaturized body size on skeletal morphology in frogs. Evolution 2002, 56, 628-641. [CrossRef] [PubMed]

22. Schwartz, C.A.; de Souza Castro, M.; Pires Júnior, O.R.; Maciel, N.M.; Schwartz, E.N.F.; Sebben, A. Princípios bioativos da pele de anfíbios: Panorama atual e perspectivas. In Herpetologia no Brasil II; Nascimento, L.B., Oliveira, M.E., Eds.; Sociedade Brasileira de Herpetologia: Belo Horizonte, Brasil, 2007; pp. 146-168. 
23. Bornschein, M.R.; Firkowski, C.R.; Belmonte-Lopes, R.; Corrêa, L.; Ribeiro, L.F.; Morato, S.A.A.; Antoniazzi, R.L., Jr.; Reinert, B.L.; Meyer, A.L.S.; Cini, F.A.; et al. Geographical and altitudinal distribution of Brachycephalus (Anura: Brachycephalidae) endemic to the Brazilian Atlantic Rainforest. PeerJ 2016, 4, e2490. [CrossRef] [PubMed]

24. Bornschein, M.R.; Teixeira, L.; Ribeiro, L.F. New record of Brachycephalus fuscolineatus Pie, Bornschein, Firkowski, Belmonte-Lopes \& Ribeiro, 2015 (Anura, Brachycephalidae) from Santa Catarina state, Brazil. Check List 2019, 15, 379-385.

25. Pie, M.R.; Ribeiro, L.F.; Confetti, A.E.; Nadaline, M.J.; Bornschein, M.R. A new species of Brachycephalus (Anura: Brachycephalidae) from southern Brazil. PeerJ 2018, 6, e5683. [CrossRef] [PubMed]

26. Ribeiro, L.F.; Bornschein, M.R.; Belmonte-Lopes, R.; Firkowski, C.R.; Morato, S.A.A.; Pie, M.R. Seven new microendemic species of Brachycephalus (Anura: Brachycephalidae) from southern Brazil. PeerJ 2015, 3, e1011. [CrossRef] [PubMed]

27. Pie, M.R.; Meyer, A.L.S.; Firkowski, C.R.; Ribeiro, L.F.; Bornschein, M.R. Understanding the mechanisms underlying the distribution of microendemic montane frogs (Brachycephalus spp., Terrarana: Brachycephalidae) in the Brazilian Atlantic Rainforest. Ecol. Model. 2013, 250, 165-176. [CrossRef]

28. Firkowski, C.R.; Bornschein, M.R.; Ribeiro, L.F.; Pie, M.R. Species delimitation, phylogeny and evolutionary demography of co-distributed, montane frogs in the southern Brazilian Atlantic Forest. Mol. Phylogenet. Evol. 2016, 100, 345-360. [CrossRef] [PubMed]

29. Pie, M.R.; Faircloth, B.C.; Bornschein, M.R.; McComarck, J.E. Phylogenomics of montane frogs of the Brazilian Atlantic Forest is consistent with isolation in sky islands followed by climatic stability. Biol. J. Linn. Soc. 2018, 125, 72-82. [CrossRef]

30. Pombal, J.P., Jr. A posição taxonômica das "variedades" de Brachycephalus ephippium (Spix, 1824) descritas por Miranda-Ribeiro, 1920 (Amphibia, Anura, Brachycephalidae). Bol. Mus. Nac. Zool. 2010, 526, 1-12.

31. Silvano, D.; Heyer, R.; Caramaschi, U. Brachycephalus nodoterga. The IUCN Red List of Threatened Species 2004: e.T54454A11149387. Available online: https://www.iucnredlist.org/species/54454/11149387 (accessed on 13 July 2019).

32. Silvano, D.; Garcia, P.; Segalla, M.V. Brachycephalus pernix. The IUCN Red List of Threatened Species 2004, e.T54455A11149530. Available online: https://www.iucnredlist.org/species/54455/11149530 (accessed on 13 July 2019).

33. Silvano, D.; Caramaschi, U. Brachycephalus hermogenesi. The IUCN Red List of Threatened Species 2010, e.T29487A9501270. Available online: https://www.iucnredlist.org/species/29487/9501270 (accessed on 13 July 2019).

34. Angulo, A. Brachycephalus ferruginus. The IUCN Red List of Threatened Species 2006, e.T135912A4229152. Available online: https://www.iucnredlist.org/species/135912/4220152 (accessed on 13 July 2019).

35. Angulo, A. Brachycephalus alipioi. The IUCN Red List of Threatened Species 2008, e.T135774A4199662. Available online: https://www.iucnredlist.org/species/135774/4199662 (accessed on 13 July 2019).

36. Angulo, A. Brachycephalus pombali. The IUCN Red List of Threatened Species 2008, e.T135830A4208137. Available online: https://www.iucnredlist.org/species/135830/4208137 (accessed on 13 July 2019).

37. Caramaschi, U.; Carvalho-e-Silva, S.P. Brachycephalus vertebralis. The IUCN Red List of Threatened Species 2004, e.T54456A11134718. Available online: https://www.iucnredlist.org/species/54456/11134718 (accessed on 13 July 2019).

38. Sluys, M.V.; Rocha, C.F. Brachycephalus ephippium. The IUCN Red List of Threatened Species 2010: e.T54453A11149233. Available online: https://www.iucnredlist.org/species/54453/11149233 (accessed on 13 July 2019).

39. Stuart, S. Brachycephalus brunneus. The IUCN Red List of Threatened Species 2006, e.T61745A12553521. Available online: https://www.iucnredlist.org/species/61746/12553521 (accessed on 13 July 2019).

40. Stuart, S. Brachycephalus izecksohni. The IUCN Red List of Threatened Species 2006, e.T61747A12553638. Available online: https://www.iucnredlist.org/species/61747/12553638 (accessed on 13 July 2019).

41. Telles, A.M.; Carvalho-e-Silva, S.P. Brachycephalus didactylus. The IUCN Red List of Threatened Species 2004, e.T54452A11148997. Available online: https://www.iucnredlist.org/species/54452/11148997 (accessed on 13 July 2019). 
42. Haddad, C.F.B.; Machado, I.F.; Giovanelli, J.G.R.; Bataus, Y.S.L.; Ublig, V.M.; Batista, F.R.Q.; Cruz, C.A.G.; Conte, C.E.; Zank, C.; Strüsmann, C.; et al. Avaliação do Risco de Extinção de Brachycephalus nodoterga Miranda-Ribeiro, 1920. In Processo de Avaliação do Risco de Extinção da Fauna Brasileira; Instituto Chico Mentes de conservação da Biodiversidade-ICMbio: Brasília, Brazil, 2016.

43. Haddad, C.F.B.; Machado, I.F.; Giovanelli, J.G.R.; Bataus, Y.S.L.; Uhlig, V.M.; Batista, F.R.Q.; Cruz, C.A.G.; Conte, C.E.; Zank, C.; Strüsmann, C.; et al. Avaliação do Risco de Extinção de Brachycephalus alipioi Pombal \& Gasparini, 2006. In Processo de Avaliação do Risco de Extinção da Fauna Brasileira; Instituto Chico Mentes de conservação da Biodiversidade-ICMbio: Brasília, Brazil, 2016.

44. Haddad, C.F.B.; Machado, I.F.; Giovanelli, J.G.R.; Bataus, Y.S.L.; Uhlig, V.M.; Batista, F.R.Q.; Cruz, C.A.G.; Conte, C.E.; Zank, C.; Strüsmann, C.; et al. Avaliação do Risco de Extinção de Brachycephalus vertebralis Pombal, 2001. In Processo de Avaliação do Risco de Extinção da Fauna Brasileira; Instituto Chico Mentes de conservação da Biodiversidade-ICMbio: Brasília, Brazil, 2016.

45. Haddad, C.F.B.; Segalla, M.V.; Bataus, Y.S.L.; Uhlig, V.M.; Batista, F.R.Q.; Garda, A.; Hudson, A.A.; Cruz, C.A.G.; Strüsmann, C.; Brasileiro, C.A.; et al. Avaliação do Risco de Extinção de Brachycephalus pernix Pombal, Wistuba \& Bornschein, 1998. In Processo de Avaliação do Risco de Extinção da Fauna Brasileira; Instituto Chico Mentes de conservação da Biodiversidade_-ICMbio: Brasília, Brazil, 2016.

46. Bland, L.M.; Colle, B.; Orme, C.D.L.; Bielby, J. Data uncertainty and the selectivity of extinction risk in freshwater invertebrates. Divers. Distrib. 2012, 18, 1211-1220. [CrossRef]

47. Morais, A.R.; Siqueira, M.N.; Lemes, P.; Maciel, N.M.; De Marco, P.; Brito, D. Unraveling the conservations status of Data Deficient species. Biol. Conserv. 2013, 166, 98-102. [CrossRef]

48. IUCN. IUCN Red List Categories and Criteria: Version 3.1., 2nd ed.; International Union for Conservation of Nature-IUCN: Gland, Switzerland; Cambridge, UK, 2012.

49. Reinert, B.L.; Bornschein, M.R.; Firkowski, C. Distribuição, tamanho populacional, hábitat e conservação do bicudinho-do-brejo Stymphalornis acutirostris Bornschein, Reinert e Teixeira, 1995 (Thamnophilidae). Rev. Bras. Ornitol. 2007, 15, 493-519.

50. Ribeiro, L.F.; Blackburn, D.C.; Stanley, E.L.; Pie, M.R.; Bornschein, M.R. Two new species of the Brachycephalus pernix group (Anura: Brachycephalidae) from the state of Paraná, southern Brazil. PeerJ 2017, 5, e3603. [CrossRef] [PubMed]

51. De Oliveira, J.C.F.; Coco, L.; Pagotto, R.V.; Pralon, E.; Vrcibradic, D.; Pombal, J.P., Jr.; Rocha, C.F.D. Amphibia, Anura, Brachycephalus didactylus (Izecksohn, 1971) and Zachaenus parvulus (Girard, 1853): Distribution extension. Check List 2012, 8, 242-244. [CrossRef]

52. Oliveira, J.C.F.; Pralon, E.; Coco, L.; Pagotto, R.V.; Rocha, C.F.D. Environmental humidity and leaf-litter depth affecting ecological parameters of a leaf-litter frog community in an Atlantic Rainforest area. J. Nat. Hist. 2013, 47, 2115-2124. [CrossRef]

53. Siqueira, C.C.; Vrcibradic, D.; Almeida-Gomes, M.; Borges, V.N.T., Jr.; Almeida-Santos, P.; Almeida-Santos, M.; Ariani, C.V.; Guedes, D.M.; Goyannes-Araújo, P.; Dorigo, T.A.; et al. Density and richness of the leaf litter frogs of an Atlantic Rainforest area in Serra dos Órgãos, Rio de Janeiro State, Brazil. Zoologia 2009, 26, 97-102. [CrossRef]

54. Almeida-Santos, M.; Siqueira, C.C.; Van Sluys, M.; Rocha, C.D.F. Ecology of the Brazilian flea frog Brachycephalus didactylus (Terrana: Brachycephalidae). J. Hepertol. 2011, 45, 251-255.

55. Siqueira, C.C.; Vrcibradic, D.; Nogueira-Costa, P.; Martins, A.R.; Dantas, L.; Gomes, V.L.R.; Bergallo, H.G.; Rocha, C.F.D. Environmental parameters affecting the structure of leaf-litter frog (Amphibia: Anura) communities in tropical forests: A case study from an Atlantic Rainforest area in southeastern Brazil. Zoologia 2014, 31, 147-152. [CrossRef]

56. Carvalho-e-Silva, A.M.T.; Silva, G.R.; Carvalho-e-Silva, S.P. Anuros da Reserva Rio das Pedras, Mangaratiba, RJ, Brasil. Biota Neotrop. 2008, 8, 199-209. [CrossRef]

57. Rocha, C.F.D.; Vrcibradic, D.; Kiefer, M.C.; Almeida-Gomes, M.; Borges-Junior, V.N.T.; Menezes, V.A.; Ariani, C.V.; Pontes, J.A.L.; Goyannes-Araújo, P.; Marra, R.V.; et al. The leaf-litter frog community from Reserva Rio das Pedras, Mangaratiba, Rio de Janeiro State, Southeastern Brazil: Species richness, composition and densities. North West. J. Zool. 2013, 9, 151-156.

58. Pombal, J.P., Jr. A new species of Brachycephalus (Anura: Brachycephalidae) from Atlantic Forest of southeastern Brazil. Amphib. Reptil. 2001, 22, 179-185. [CrossRef] 
59. Ribeiro, L.F.; Alves, A.C.R.; Haddad, C.F.B. Two new species of Brachycephalus Günther, 1858 from the state of Paraná, southern Brazil (Amphibia, Anura, Brachycephalidae). Bol. Mus. Nac. Zool. 2005, 519, 1-18.

60. Alves, A.C.R.; Ribeiro, L.F.; Haddad, C.F.B.; Reis, S.F. Two new species of Brachycephalus (Anura: Brachycephalidae) from the Atlantic Forest in Paraná State, southern Brasil. Hepertologica 2006, 62, 221-233. [CrossRef]

61. Alves, A.C.R.; Sawaya, R.J.; Reis, S.F.; Haddad, C.F.B. New species of Brachycephalus (Anura: Brachycephalidae) from the Atlantic Rain Forest in São Paulo State, Southeastern Brazil. J. Hepertol. 2009, 43, 212-219. [CrossRef]

62. Da Silva, H.R.; Campos, L.A.; Sebben, A. The auditory region of Brachycephalus and its bearing on the monophyly of the genus (Anura: Brachycephalidae). Zootaxa 2007, 1422, 59-68. [CrossRef]

63. Verdade, V.K.; Rodrigues, M.T.; Cassimiro, J.; Pavan, D.; Liou, N.; Lange, M. Advertisement call, vocal activity, and geographic distribution of Brachycephalus hermogenesi (Giaretta and Sawaya, 1998) (Anura, Brachycephalidae). J. Herpetol. 2008, 42, 542-549. [CrossRef]

64. Clemente-Carvalho, R.G.B.; Antoniazzi, M.M.; Jared, C.; Haddad, C.F.B.; Alvez, A.C.R.; Rocha, H.S.; Pereira, G.R.; Oliveira, D.F.; Lopes, R.T.; Reis, S.F. Hyperossification in miniaturized toadlets of the genus Brachycephalus (Amphibia: Anura: Brachycephalidae): Microscopic structure and macroscopic patterns of variation. J. Morphol. 2009, 270, 1285-1295. [CrossRef] [PubMed]

65. Campos, L.A. Sistemática Filogenética do Gênero Brachycephalus Ftzinger, 1826 (Anura Brachycephalidae) Com Base Em Dados Morfológicos. Ph.D. Thesis, Universidade de Brasília, Brasília, Brazil, 2011.

66. Pombal, J.P., Jr.; Izecksohn, E. Uma nova espécie de Brachycephalus (Anura, Brachycephalidae) do Estado do Rio de Janeiro. Pap. Av. Zool. 2011, 51, 443-451. [CrossRef]

67. Siqueira, C.C.; Vrcibradic, D.; Dorigo, T.A.; Rocha, C.F.D. Anurans from two high-elevation areas of Atlantic Forest in the state of Rio de Janeiro, Brazil. Zoologia 2011, 28, 457-464. [CrossRef]

68. Rocha, C.F.D.; Van Sluys, M.; Alves, M.A.S.; Bergallo, H.G.; Vrcibradic, D. Activity of leaf-litter frogs: When should frogs be sampled? J. Herpetol. 2000, 34, 285-287. [CrossRef]

69. Rocha, C.F.D.; Van Sluys, M.; Alves, M.A.S.; Bergallo, H.G.; Vrcibradic, D. Estimates of forest floor litter frog communities: A comparison of two methods. Austral. Ecol. 2001, 26, 14-21. [CrossRef]

70. Van Sluys, M.; Vrcibradic, D.; Alves, M.A.S.; Bergallo, H.G.; Rocha, C.F.D. Ecological parameters of the leaf-litter frog community of an Atlantic Rainforest area at Ilha Grande, Rio de Janeiro state, Brazil. Austral. Ecol. 2007, 32, 254-260. [CrossRef]

71. Pimenta, B.V.S.; Bérnils, R.S.; Pombal, J.P., Jr. Amphibia, Anura, Brachycephalidae, Brachycephalus hermogenesi: Filling gap and geographic distribution map. Check List 2007, 3, 277-279. [CrossRef]

72. Condez, T.H.; Monteiro, J.P.C.; Comitti, E.J.; Garcia, P.C.A.; Amaral, I.B.; Haddad, C.F.B. A new species of flea-toad (Anura: Brachycephalidae) from southern Atlantic Forest, Brazil. Zootaxa 2016, 4083, 40-56. [CrossRef]

73. Firkowski, C.R. Diversification and microendemism in montane refugia from the Brazilian Atlantic Forest. Master's Thesis, Universidade Federal do Paraná, Curitiba, Brazil, 2013.

74. Bornschein, M.R.; Ribeiro, L.F.; Blackburn, D.C.; Stanley, E.L.; Pie, M.R. A new species of Brachycephalus (Anura: Brachycephalidae) from Santa Catarina, southern Brazil. PeerJ 2016, 4, e2629. [CrossRef]

75. Monteiro, J.P.C.; Condez, T.H.; Garcia, P.C.A.; Comitti, E.J.; Amaral, I.B.; Haddad, C.F.B. A new species of Brachycephalus (Anura, Brachycephalidae) from the coast of Santa Catarina State, southern Atlantic Forest, Brazil. Zootaxa 2018, 4407, 483-505. [CrossRef]

76. Teixeira, L.; Ribeiro, L.F.; Côrrea, L.; Confetti, A.E.; Pie, M.R.; Bornschein, M.R. A second record of the recently described Brachycephalus albolineatus Bornschein, Ribeiro, Blackburn, Stanley \& Pie, 2016 (Anura, Brachycephalidae). Check List 2018, 14, 1013-1016.

77. Pereira, M.S.; Candaten, A.; Milani, D.; Oliveira, F.B.; Gardelin, J.; Rocha, C.F.D.; Vrcibradic, D. Geographic distribution: Brachycephalus hermogenesi. Herpetol. Rev. 2010, 41, 506.

78. Santos-Pereira, M.; Candaten, A.; Milani, D.; Oliveira, F.B.; Gardelin, J.; Rocha, C.F.D. Seasonal variation in the leaf-litter frog community (Amphibia: Anura) from an Atlantic Forest area in the Salto Morato Natural Reserve, southern Brazil. Zoologia 2011, 28, 755-761. [CrossRef]

79. Santos-Pereira, M.; Milani, D.; Barata-Bittencourt, L.F.; Iapp, T.M.; Rocha, C.F.D. Anuran species of the Salto Morato Nature Reserve in Paraná, southern Brazil: Review of the species list. Check List 2016, 12, 1907. [CrossRef] 
80. Pombal, J.P., Jr.; Gasparini, J.L. A new Brachycephalus (Anura: Brachycephalidae) from the Atlantic Rainforest of Espírito Santo, southeastern Brazil. S. Am. J. Herpet. 2006, 1, 87-93. [CrossRef]

81. Clemente-Carvalho, R.G.B.; Klaczko, J.; Perez, S.R.; Alves, A.C.R.; Haddad, C.F.B.; Reis, S.F. Molecular phylogenetic relationships and phenotypic diversity in miniaturized toadlets, genus Brachycephalus (Amphibia: Anura: Brahycephalidae). Mol. Phylogenet. Evol. 2011, 61, 79-89. [CrossRef]

82. Clemente-Carvalho, R.B.G.; Giaretta, A.A.; Condez, T.H.; Haddad, C.F.B.; Reis, S.F. A new species of miniaturized toadlet, genus Brachycephalus (Anura: Brachycephalidae), from the Atlantic Forest of southeastern Brazil. Herpetologica 2012, 68, 365-374. [CrossRef]

83. Miranda-Ribeiro, A. Os Brachycephalideos do Museu Paulista (com tres especies novas). Rev. Mus. Paulista 1920, 12, 306-318.

84. Condez, T.H.; Clemente-Carvalho, R.B.G.; Haddad, C.F.B.; Reis, S.F. A new species of Brachycephalus (Anura: Brachycephalidae) from the highlands of the Atlantic Forest, southeastern Brazil. Herpetologica 2014, 70 , 89-99. [CrossRef]

85. Moura, M.R.; Motta, A.P.; Fernandes, V.D.; Feio, R.N. Herpetofauna from Serra do Brigadeiro, an Atlantic Forest remain in the state of Minas Gerais, southeastern Brazil. Biota Neotrop. 2012, 12, 209-235. [CrossRef]

86. Guimarães, C.S.; Luz, S.; Rocha, P.C.; Feio, R.N. The dark side of pumpkin toadlet: A new species of Brachycephalus (Anura: Brachycephalidae) from Serra do Brigadeiro, southeastern Brazil. Zootaxa 2017, 4258, 327-344. [CrossRef]

87. Pombal, J.P., Jr.; Sazima, I.; Haddad, C.F.B. Breeding behavior of the pumpkin toadlet, Brachycephalus ephippium (Brachycephalidae). J. Herpetol. 1994, 28, 516-519. [CrossRef]

88. Giaretta, A.A. Diversidade e Densidade de Anuros de Serapilheira Num Gradiente Altitudinal na Mata Atlântica Costeira. Ph.D. Thesis, Universidade Estadual de Campinas, Campinas, Brazil, 1999.

89. Giaretta, A.A.; Facure, K.G.; Sawaya, R.J.; Meyer, J.H.M.; Chemin, N. Diversity and abundance of litter frogs in a montane forest of Southeastern Brazil: Seasonal and altitudinal changes. Biotropica 1999, 31, 669-674. [CrossRef]

90. Giaretta, A.A.; Sawaya, R.J.; Machado, G.; Araújo, M.S.; Facure, K.G.; Medeiros, H.F.; Nunes, R. Diversity and abundance of litter frogs at altitudinal sites at Serra do Japi, Southeastern Brazil. Rev. Brasil. Zool. 1997, 14, 341-346. [CrossRef]

91. Clemente-Carvalho, R.B.G.; Monteiro, L.R.; Bonato, V.; Rocha, H.S.; Pereira, G.R.; Oliveira, D.F.; Lopes, R.T.; Haddad, C.F.B.; Martins, E.G.; Reis, S.F. Geographic variation in cranial shape in the Pumpkin Toadlet (Brachycephalus ephippium): A geometric analysis. J. Herpetol. 2008, 42, 176-185. [CrossRef]

92. Clemente-Carvalho, R.G.B.; Alves, A.C.R.; Perez, S.I.; Haddad, C.F.B.; Reis, S.F. Morphological and molecular variation in the Pumpkin Toadlet Branchycephalus ephippium (Anura: Brachycephalidae). J. Herpetol. 2011, 45, 94-99. [CrossRef]

93. Zaher, H.; Aguiar, E.; Pombal, J.P. Paratelmatobius gaigeae (Cochran, 1938) rediscovered (Amphibia, Anura, Leptodactylidae). Arquiv. Mus. Nac. 2005, 63, 321-328.

94. Dixo, M.; Verdade, V.K. Herpetofauna de serrapilheira da Reserva Florestal de Morro Grande, Cotia (SP). Biota Neotrop. 2006, 6, 1-20. [CrossRef]

95. Siqueira, C.C.; Vrcibradic, D.; Rocha, C.F.D. Altitudinal records of data-deficient and threatened frog species from the Atlantic Rainforest of the Serra dos Órgãos mountains, in southeastern Brazil. Braz. J. Biol. 2013, 73, 229-230. [CrossRef]

96. Dorigo, T.A.; Siqueira, C.C.; Vrcibradic, D.; Maia-Carneiro, T.; Almeida-Santos, M.; Rocha, C.F.D. Ecological aspects of the pumpkin toadlet, Brachycephalus garbeanus Miranda-Ribeiro, 1920 (Anura: Neobatrachia: Brachycephalidae), in a highland forest of southeastern Brazil. J. Nat. Hist. 2012, 46, 2497-2507. [CrossRef]

97. Pombal, J.P., Jr.; Wistuba, E.M.; Bornschein, M.R. A new species of brachycephalid (Anura) from the Atlantic Rain Forest of Brazil. J. Herpetol. 1998, 32, 70-74. [CrossRef]

98. Haddad, C.F.B.; Alves, A.C.R.; Clemente-Carvalho, R.B.G.; Reis, S.F. A new species of Brachycephalus from the Atlantic Rain Forest in São Paulo state, southeastern Brazil (Amphibia: Anura: Brachycephalidae). Copeia 2010, 410-420. [CrossRef]

99. Abegg, A.D.; Ortiz, F.R.; Rocha, B.; Condes, T.H. A new record for Brachycephalus nodoterga (Amphibia, Anura Brachycephalidae) in the state of São Paulo, Brazil. Check List 2015, 11, 1769. [CrossRef] 
100. Ribeiro, R.S. Ecologia Alimentar das Quatro Espécies Dominantes da Anurofauna de Serapilheira Em Um Gradiente Altitudinal na Ilha de São Sebastião, SP. Master Thesis, Universidade Estadual Paulista “Júlio de Mesquita Filho", Rio Claro, São Paulo, Brazil, 2006.

101. Campos, L.A.; Silva, H.R.; Sebben, A. Morphology and development of additional bony elements in the genus Brachycephalus (Anura: Brachycephalidae). Biol. J. Linn. Soc. 2010, 99, 752-767. [CrossRef]

102. Oliveira, E.G. História Natural de Brachycephalus pitanga no Núcleo Santa Virgínia, Parque Estadual da Serra do Mar, Estado de São Paulo. Master Thesis, Universidade Estadual Paulista "Júlio de Mesquita Filho", Rio Claro, Brazil, 2013.

103. Tandel, M.C.F.F.; Loibel, S.; Oliveira, E.G.; Haddad, C.F.B. Diferenciação de 3 tipos de vocalizações (cantos) na espécie Brachycephalus pitanga. Revista da Estatística da Universidade Federal de Ouro Preto 2014, 3, 374-386.

104. Araújo, C.B.; Guerra, T.J.; Amatuzzi, M.C.O.; Campos, L.A. Advertisement and territorial calls of Brachycephalus pitanga (Anura: Brachycephalidae). Zootaxa 2012, 3302, 66-67. [CrossRef]

105. Bornschein, M.R.; Ribeiro, L.F.; Rollo, M.M., Jr.; Confetti, A.E.; Pie, M.R. Advertisement call of Brachycephalus albolineatus (Anura: Brachycephalidae). PeerJ 2018, 6, e5273. [CrossRef]

106. Fontoura, P.L.; Ribeiro, L.F.; Pie, M.R. Diet of Brachycephalus brunneus (Anura: Brachycephalidae) in the Atlantic Rainforest of Paraná, southern Brazil. Zoologia 2011, 28, 687-689. [CrossRef]

107. Pie, M.R.; Ströher, P.R.; Bornschein, M.R.; Ribeiro, L.F.; Faircloth, B.C.; Mccormack, J.E. The mitochondrial genome of Brachycephalus brunneus (Anura: Brachycephalidae), with comments on the phylogenetic position of Brachycephalidae. Biochem. Syst. Ecol. 2017, 71, 26-31. [CrossRef]

108. Monteiro, J.P.C.; Condez, T.H.; Garcia, P.C.A.; Haddad, C.F.B. The advertisement calls of two species of Brachycephalus (Anura: Brachycephalidae) from southern Atlantic Forest, Brazil. Zootaxa 2018, 4415, 183-188. [CrossRef]

109. Wistuba, E.M. História Natural de Brachycephalus pernix Pombal, Wistuba e Bornschein, 1998 (Anura) no Morro Anhangava, Município de Quatro Barras, Estado do Paraná. Ph.D. Thesis, Universidade Federal do Paraná, Curitiba, Brazil, 1998.

110. Pires, O.R., Jr.; Sebben, A.; Schwartz, E.F.; Morales, R.A.V.; Bloch, C., Jr.; Schwartz, C.A. Further report of the occurrence of tetrodotoxin and new analogues in the Anuran family Brachycephalidae. Toxicon 2005, 45, 73-79. [CrossRef] [PubMed]

111. Ribeiro, L.F.; Ströher, P.R.; Firkowski, C.R.; Cini, F.A.; Bornschein, M.R.; Pie, M.R. Brachycephalus pernix (Anura: Brachycephalidae), a new host of Ophiotaenia (Eucestoda: Proteocephalidea). Herpetol. Notes 2014, 7, 291-294.

112. Pie, M.R.; Ribeiro, L.F. A new species of Brachycephalus (Anura: Brachycephalidae) from the Quiriri mountain range of southern Brazil. PeerJ 2015, 3, e1179. [CrossRef] [PubMed]

113. Garey, M.V.; Lima, A.M.X.; Hartmann, M.T.; Haddad, C.F.B. A new species of miniaturized toadlet, genus Brachycephalus (Anura: Brachycephalidae), from southern Brazil. Herpetologica 2012, 68, 266-271. [CrossRef]

114. Bornschein, M.R.; Rollo, M.M., Jr.; Pie, M.R.; Confetti, A.E.; Ribeiro, L.F. Redescription of the advertisement call of Brachycephalus tridactylus (Anura: Brachycephalidae). Phyllomedusa 2019, 18, 3-12. [CrossRef]

115. Cunha, A.K.; Oliveira, I.S.; Hartmann, M.T. Anurofauna da Colônia Castelhanos, na Área de Proteção Ambiental de Guaratuba, Serra do Mar paranaense, Brasil. Biotemas 2010, 23, 123-134.

116. De Oliveira, A.K.C.; Oliveira, I.S.; Hartmann, M.T.; Silva, N.R.; Toledo, L.F. Amphibia, Anura, Brachycephalidae, Brachycephalus hermogenesi (Giaretta and Sawaya, 1998): New species record in the state of Paraná, southern Brazil and geographic distribution map. Check List 2011, 7, 17-18. [CrossRef]

117. Mariotto, L.R. Anfíbios de Um Gradiente Altitudinal Em Mata Atlântica. Master Thesis, Universidade Federal do Paraná, Curitiba, Brazil, 2014.

118. Condez, T.H.; Sawaya, R.J.; Dixo, M. Herpetofauna dos remanescentes de Mata Atlântica da região de Tapiraí e Piedade, SP, sudeste do Brasil. Biota Neotrop. 2009, 9, 157-185. [CrossRef]

119. Verdade, V.K.; Rodrigues, M.T.; Pavan, D. Anfíbios Anuros da Região da Estação Biológica do Alto da Serra de Paranapiacaba. Patrimônio da Reserva Biológica do Alto da Serra de Paranapiacaba: A antiga Estação Biológica do Alto da Serra; Governo do Estado de São Paulo, Secretaria do Meio Ambiente: São Paulo, Brazil, 2009; pp. 579-603.

120. Trevine, V.; Forlani, M.C.; Haddad, C.F.B.; Zaher, H. Herpetofauna of Paranapiacaba: Expanding our knowledge on a historical region in the Atlantic forest of southeastern Brazil. Zoologia 2014, 31, 126-146. [CrossRef] 
121. Collins, J.P. Amphibian decline and extinction: What we know and what we need to learn. Dis. Aquat. Organ. 2010, 92, 93-99. [CrossRef]

122. Walter, H. Zonas de Vegetación y Clima: Breve Exposición Desde el Punto de Vista Causal y Global; Omega: Barcelona, Espanha, 1977; pp. 1-245.

123. Walsh, R.P.D. Climate. In The Tropical Rain Forest; Richards, P.W., Ed.; Cambridge University Press: Cambridge, UK, 1979; pp. 159-205.

124. Roderjan, C.V. O Gradiente da Floresta Ombrófila Densa no Morro Anhangava, Quatro Barras, PR-Aspectos Climáticos, Pedológicos e Fitossociológicos. Ph.D. Thesis, Universidade Federal do Paraná, Curitiba, Brazil, 1994.

125. Pimm, S.L.; Jenkins, C.N.; Abell, R.; Brooks, T.M.; Gittleman, J.L.; Joppa, L.N.; Raven, P.H.; Roberts, C.M.; Sexton, J.O. The biodiversity of species and their rates of extinction, distribution, and protection. Science 2014, 344, 1246752. [CrossRef] [PubMed]

126. Bornschein, M.R.; Firkowski, C.R.; Baldo, D.; Ribeiro, L.F.; Belmonte-Lopes, R.; Corrêa, L.; Morato, S.A.A.; Pie, M.R. Three new species of phytotelm-breeding Melanophryniscus from the Atlantic Rainforest of southern Brazil (Anura: Bufonidae). PLoS ONE 2015, 10, e0142791. [CrossRef] [PubMed]

127. Nadaline, M.J.; Ribeiro, L.F.; Teixeira, L.; Vannuchi, F.S.; Bornschein, M.R. New record of Melanophryniscus biancae Bornschein, Baldo, Pie, Firkowski, Ribeiro \& Corrêa, 2015 (Anura: Bufonidae) from Paraná, Brazil, with comments on its phytotelm-breeding ecology. Check List, in press.

(C) 2019 by the authors. Licensee MDPI, Basel, Switzerland. This article is an open access article distributed under the terms and conditions of the Creative Commons Attribution (CC BY) license (http://creativecommons.org/licenses/by/4.0/). 